\title{
Design optimization of offshore wind farms with multiple types of wind turbines
}

\author{
Feng, Ju; Shen, Wen Zhong
}

Published in:

Applied Energy

Link to article, DOI:

10.1016/j.apenergy.2017.08.107

Publication date:

2017

Document Version

Peer reviewed version

Link back to DTU Orbit

Citation (APA):

Feng, J., \& Shen, W. Z. (2017). Design optimization of offshore wind farms with multiple types of wind turbines. Applied Energy, 205, 1283-1297. https://doi.org/10.1016/j.apenergy.2017.08.107

\section{General rights}

Copyright and moral rights for the publications made accessible in the public portal are retained by the authors and/or other copyright owners and it is a condition of accessing publications that users recognise and abide by the legal requirements associated with these rights.

- Users may download and print one copy of any publication from the public portal for the purpose of private study or research.

- You may not further distribute the material or use it for any profit-making activity or commercial gain

- You may freely distribute the URL identifying the publication in the public portal

If you believe that this document breaches copyright please contact us providing details, and we will remove access to the work immediately and investigate your claim. 


\title{
Design optimization of offshore wind farms with multiple types of wind turbines
}

\author{
Ju Feng, Wen Zhong Shen* \\ Department of Wind Energy, Technical University of Denmark, DK-2800 Lyngby, Denmark
}

\begin{abstract}
Most studies on offshore wind farm design assume a uniform wind farm, which consists of an identical type of wind turbines. In order to further reduce the cost of energy, we investigate the design of non-uniform offshore wind farms, i.e., wind farms with multiple types of wind turbines and hub-heights. Given a set of different types of wind turbines with a different default hub height for each type, we can specify the design of a wind farm by the types of turbines, number of turbines for each type, and turbine locations. We consider the optimization of such design to minimize the levelized cost of energy, which is calculated using a capital cost model that covers the turbine cost and the balance of plant cost. An empirical wind turbine design cost and scaling model is utilized to model the cost of turbines with different sizes. Constraints on wind farm boundary, wind turbine proximity and total capacity are also included. We solve the problem with a newly developed extended random search algorithm and tested it in a realistic design optimization problem based on the Horns Rev 1 offshore wind farm in Denmark. The optimized non-uniform designs are compared with their uniform counterparts. We find that a non-uniform design can achieve a lower levelized cost of energy than its uniform counterparts, when the capital cost per MW is slightly lower for the smaller size turbine. Comparison with the mixed-discrete particle swarm optimization algorithm is also carried out for a non-uniform wind farm design problem with fixed number of turbines, which shows the effectiveness and superiority of the proposed algorithm. Finally, the advantages and possible disadvantages of non-uniform design are also identified and discussed.
\end{abstract}

Keyords: offshore wind farm; non-uniform wind farm; layout optimization; design optimization; random search algorithm; levelized cost of energy

\section{Introduction}

The world's first offshore wind farm (WF), Vindeby offshore WF located in Denmark, was connected to the grid in 1991. After more than 25 years of service, its owner and operator, DONG Energy has recently decided to decommission it [1]. This event reminds us that offshore wind energy is still a young field with a short history. Nevertheless, offshore wind energy has achieved an astonishing

\footnotetext{
${ }^{*}$ Corresponding author. Tel.: +45 45254317;

E-mail address: wzsh@dtu.dk(W.Z.Shen).
} 
development in the recent years. According to the latest statistics from the Global Wind Energy Council (GWEC), the global cumulative offshore wind capacity was increased from 4117MW in 2011 to $14384 \mathrm{MW}$ in 2016 , representing an average annual increase of $28.4 \%$ [2]. As the main form of utilizing offshore wind energy, offshore WFs proliferate in the world in the past two decades, especially in Europe. Consequently, the design and planning problem of offshore WFs has received a lot of attention [3].

\subsection{Traditional offshore WF layout optimization}

Among many problems in WF design and planning, layout optimization is one of central importance, which is to find the optimal positions of wind turbines (WTs) inside an WF with regards to a single/multiple objective(s), while satisfying certain constraints and assumptions [4]. Since the seminal work of Mosetti et al. in 1994 [5], this problem has been investigated by a large amount of studies, with the majority focusing on onshore WFs [6].

Many recent studies have also investigated the specific problem of offshore WF layout optimization [7]. For example, Elkinton el al. [8] developed a tool for optimizing offshore WF layout to minimize the levelized production cost (LPC), considering simple cost models for different components and using genetic algorithm (GA) and greedy heuristic algorithm; Rivas et al. [9] applied simulated annealing to optimize an offshore WF layout, with annual energy production (AEP) as its objective function; González et al. [10] proposed to optimize offshore WF layout for maximizing its net present value (NPV), by means of an improved GA with tailored operators; Gao et al. [11,12] investigated the potential and feasibility of constructing offshore WFs in Hong Kong, by optimizing the offshore WF layout for minimal cost of energy (COE) with the multi-population GA; Salcedo-Sanz et al. [13] applied the Coral Reefs Optimization algorithm to maximize the AEP of offshore WFs; Feng et al. [14] developed a new heuristic global search algorithm, Random Search (RS) algorithm, and applied it in the layout optimization of the Horns Rev 1 offshore WF in Denmark for maximizing AEP; Gao et al. [15] proposed a two-dimensional wake model, Jensen-Gaussian wake model, and applied it in WF layout optimization using multi-population GA; layout optimization with regards to the robustness of WF power production in the changing wind was also investigated in a recent study [16]; González et al. [17] studied the optimization problem of offshore WFs with grid-like layouts. While most of the published studies, including those mentioned above, applied various metaheuristic algorithms in the optimization problems, there are several efforts made recently to apply conventional mathematical optimization techniques, such as sequential convex programming [18], nonlinear mathematical programming [19,20] and mixed integer programming method [21].

Since most of the constructed offshore WFs are uniform, i.e., they are composed of one type of 
WTs with the same hub-height, the majority of the published studies focused on uniform offshore WFs. Usually a fixed type of WT was considered in these studies, while a few considered the problem of type selection, i.e., choosing a proper type from a group of available WT types. For instance, Mustakerov et al. [22] addressed the WT type and number choice optimization problem for minimizing the cost per unit energy. In their study, the cost model depends only on the number of WTs, and they assumed an array-like rectangular WF with the numbers of WTs per row and per column as design variables. They then solved the problem with the combinatorial optimization method for multiple types of WTs. Their results showed that using big size WTs is more profitable than using small size WTs. However, their study suffered from the simplified wind cases and a lack of proper wake effect modelling.

In another study, Chowdhury et al. [23] formulated three different scenarios for WF design: optimizing WF layout with defined WT type; simultaneously optimizing the WF layout and selecting a single type of WTs; simultaneously optimizing the WF layout and the type of each WT. In the second scenario, they assumed that WF consists of a fixed number of identical WTs and solved it with a mixed-discrete particle swarm optimization (PSO) algorithm [24]. They tested the methodology for a 25 turbine WF in North Dakota, USA and improved the WF capacity factor by $6.4 \%$ when simultaneously optimizing the layout and the turbine type selection. Note that the capacity factor is defined by the average power generated by WF, divided by its total capacity.

Furthermore, it should be noted that the complete design of an offshore WF is more than the type selection and siting of WTs. Many other factors (such as the design of foundations, electrical systems) and requirements/considerations (e.g. visual impact [25] and underwater noise emission [26]) are also parts of the complete design and in principle should be taken into consideration. Several studies tried to solve the integrated optimization problem of both the layout of WTs and other factors for uniform offshore WFs. For example, the WF layout and the internal cable network were optimized simultaneously, both as a multi-objective optimization problem in [27] and as a single-objective optimization problem in [28]. Guirguis et al. [20] proposed a gradient-based approach to solve the multi-objective WF design optimization problems considering land footprint, energy output, electrical infrastructure and environmental impact. Studies of solving the design optimization problems with other factors were also done, usually by assuming the WF layout is given. For example, the optimization of offshore WF electrical system design was studied in [29] and many other studies as summarized in the review article [30].

\subsection{Non-uniform offshore WF optimization}

Constructing a uniform offshore WF with one type of WTs seems to be a good choice, as this kind of 
design naturally brings convenience for supply chain management, installation, operation and maintenance (O\&M). Nevertheless, we should realize that there are no fundamental limits preventing us from using multiple types of WTs in offshore WFs. When limiting the offshore WF design to using only one type of WTs, we are essentially limiting ourselves in the feasible design space, leaving a large part of the design space unexplored. Thus, it is beneficial to at least consider the option of nonuniform offshore WF in the design and planning stage. Here we use the term 'non-uniform offshore WF' to represent an offshore WF that is composed of multiple types of WTs.

However, most of the published studies on WF design optimization assumed that one type of WTs are used, while few recent studies considered the possibility of non-uniform WFs. One example is the study done by Chowdhury et al. in 2012 [31]. Among the 3 different layout optimization cases in this study, the second one considered WF with non-identical WTs (multiple WT types). The optimization problem was formulated to find the locations and types for each WT that maximizes the WF's capacity factor, with constraints on WF boundary, minimal clearance distance between WTs and maximal WF cost. The WF cost was calculated in considering each WT's cost, which was in turn estimated based on a quadratic function of the WT's rotor diameter. They solved the problem using a constrained PSO and found that an optimal combination of different types of WTs could appreciably improve the WF capacity factor. Although it is a seminal work that first considered non-uniform WF, this study [31] have several drawbacks: (i) the experimental WTs used in the study have quite different power curves from today's mainstream MW sized WTs; (ii) the cost model used is quite simple and not included in the objective function; (iii) the wind condition considered (unidirectional and fixed wind speed) is too simplified to reflect the realistic situation in WF design; (iv) the rotor diameters of different types of WTs are treated as continuous variables; (v) the choice of the proper number of WTs is not taken into consideration. In the scenario 3 of a later study also done by Chowdhury et al. [23], which we briefly described before, the first four of these drawbacks were largely tackled. In this study, the design variables include $x$ and $y$ coordinates (continuous variables) and turbine type codes (discrete variables) for a given number of WTs.

The potential benefits of non-uniform WFs have also been investigated by Chamorro et al. [32] through wind tunnel experiments. They placed a non-uniform WF composed of 3 by 8 model WTs with 2 alternating sizes in a boundary layer flow over both a smooth and rough surfaces and focused on investigating the dynamics involved in a non-uniform WF and the effect of surface roughness. Their results showed that reduced levels of turbulence and increased levels of kinetic energy flux to the WF can be achieved using variable-size WTs in a WF, suggesting the potential advantages of nonuniform WF design.

More recently, Abdulrahman et al. [33] investigated WF design optimization considering WT type 
selection and hub height variation for three different objective functions: power, capacity factor and COE. They considered two cases in this study: the first one optimizes the locations, types and hubheights of 6 WTs in a line; the second one optimizes the types and hub-heights of 18 WTs with fixed locations. They assumed a fixed wind speed blow along a given wind direction to simplify the study and solved the optimization problem using GA. Their study also demonstrated the benefits of using non-uniform design for offshore WFs.

A group of studies have also investigated the WF design of single type of WTs with different hub heights: Chen et al. [34] consider the design optimization of WF composed of GE 1.6MW WTs with 2 different hub heights $(80$ and $100 \mathrm{~m})$, including the turbine locations and hub heights optimization solved with GA; Chen et al. [35] solved the similarly formulated problem (single type of WT, 2 hub heights) using a three-dimensional greedy algorithm; Vasel-Be-Hagh et al. [36] studied the hub height optimization problem of WFs, with the hub heights of each WT as design variable and all other parameters (such as WT type, WT locations) fixed. All these studies suggest using multiple hub heights can lead to higher power production comparing to the single hub height counterparts.

Generally speaking, the design of a non-uniform offshore WF needs to at least specify the number of WTs (integer variable), WT's type (discrete variable) and location coordinates (continuous variables). Since the optimization problem of such non-uniform offshore WFs is beyond the scope of the traditional WF layout optimization, we use the term 'design optimization' in this work. Due to the nature of the design variables, it is a constrained mixed integer-discrete-continuous nonlinear optimization problem and is generally difficult to solve, except using metaheuristics such as differential evolution [37] or the mixed-discrete PSO [24].

As mentioned before, the complete design of an offshore WF includes other factors beyond the definition given above. In this study, we choose not to include these factors as design variables in the optimization, but instead only consider the costs related to some of them in the capital cost model. This is mainly due to the lack of accurate cost models for these factors that can be incorporated in a simultaneous optimization of the complete design. We assume, after the design optimization involving all WTs is conducted, one/several second stage optimization process(es) can be done for the design of these other factors.

\subsection{Contributions of this study}

We investigate the design optimization problem of non-uniform offshore WFs in a realistic setting, by considering an unfixed number of multiple types of WTs in a given offshore WF. These WT types have different rotor sizes, rated power values and hub heights. The objective function we choose is the levelized cost of energy (LCOE), with constraints on WF boundary, wind turbine proximity and total 
capacity. An extended version of the RS algorithm [14] is proposed to solve the design optimization problem. This method is then tested in a realistic non-uniform offshore WF design problem, which is based on the real wind characteristics at the Horns Rev 1 offshore WF in Denmark, three types of WTs and an empirical WT design cost and scaling model. The result of the non-uniform design of this offshore WF is compared with its uniform counterparts, and the advantages and possible disadvantages of non-uniform design are identified and discussed. Comparison between the proposed algorithm and the mixed-discrete PSO is also conducted for a test case with simple wind condition. The main contributions of this study can be summarized as follows:

- The design optimization problem of non-uniform offshore WFs to minimize LCOE is formulated and solved under realistic scenarios;

- An effective metaheuristic algorithm is developed to solve the related constrained mixedinteger-discrete-continuous nonlinear optimization problems;

- The developed algorithm is effective, robust, easy to implement and capable of finding the final optimal design, whether it is uniform or non-uniform;

- The developed algorithm achieves better performance than the mixed-discrete PSO algorithm for a non-uniform WF design test case;

- It is found that whether to use large WTs alone, small WTs alone or multiple types of WTs depends strongly on the capital costs of different WT types.

\section{Selection of wind turbines}

The size of WTs in offshore WFs has grown considerably in recent years. The first offshore WF in the world, Vindeby, had a total capacity of $4.95 \mathrm{MW}$ composed of 11 Bonus $450 \mathrm{~kW}$ WTs [1], while the world's largest offshore WF today, the London Array, was commissioned in 2012 and has a total capacity of 630MW with 175 Siemens 3.6MW WTs [38]. More recently, 32 MHI Vestas V164 8MW WTs have been installed in the Burbo Bank Extension offshore WF in UK, representing the first commercial offshore installation of the world's largest WTs [39]. In fact, we could expect to see even larger WTs in the future offshore WFs, as the feasibility of upscaling WT size up to 20MW has already been confirmed by researchers [40]. As a consequence, today's WF developers face a range of choices with different sized WTs, typically in the scale of 1-10MW, enabling them to install multiple types of WTs in a WF.

In order to limit the complexity for installation and O\&M, we assume the number of different WT types installed in a given offshore WF should be limited and consider only 3 types of WTs with different sizes. The largest WT is the LEANWIND 8MW turbine (LW8), which is a reference turbine defined primarily based on the published data relating to the MHI Vestas V164 8MW turbine in the 
EU project LEANWIND [41]. The other two types of WTs are downscaled from LW8, which have a rated power of $5 \mathrm{MW}$ and $2 \mathrm{MW}$. The parameters and characteristics of these three types of WTs are given in Table 1 and Figure 1. The detailed parameters for power and thrust characteristics are presented in Table A1 of the Appendix. Note that the thrust coefficient curves for the 3 types of WTs are identical, as shown in Table A1.

Table 1. General parameters of the three types of WTs

\begin{tabular}{cccc}
\hline Name & Rated power [MW] & Rotor diameter [m] & Hub height [m] \\
\hline LW8 & 8 & 164 & 110 \\
LW5 & 5 & 130 & 90 \\
LW2 & 2 & 82 & 70 \\
\hline Note: the cut-in, rated and cut-out wind speeds for the three types of \\
WTs are the same, which are $4 \mathrm{~m} / \mathrm{s}, 12.5 \mathrm{~m} / \mathrm{s}$ and $25 \mathrm{~m} / \mathrm{s}$. \\
\hline
\end{tabular}
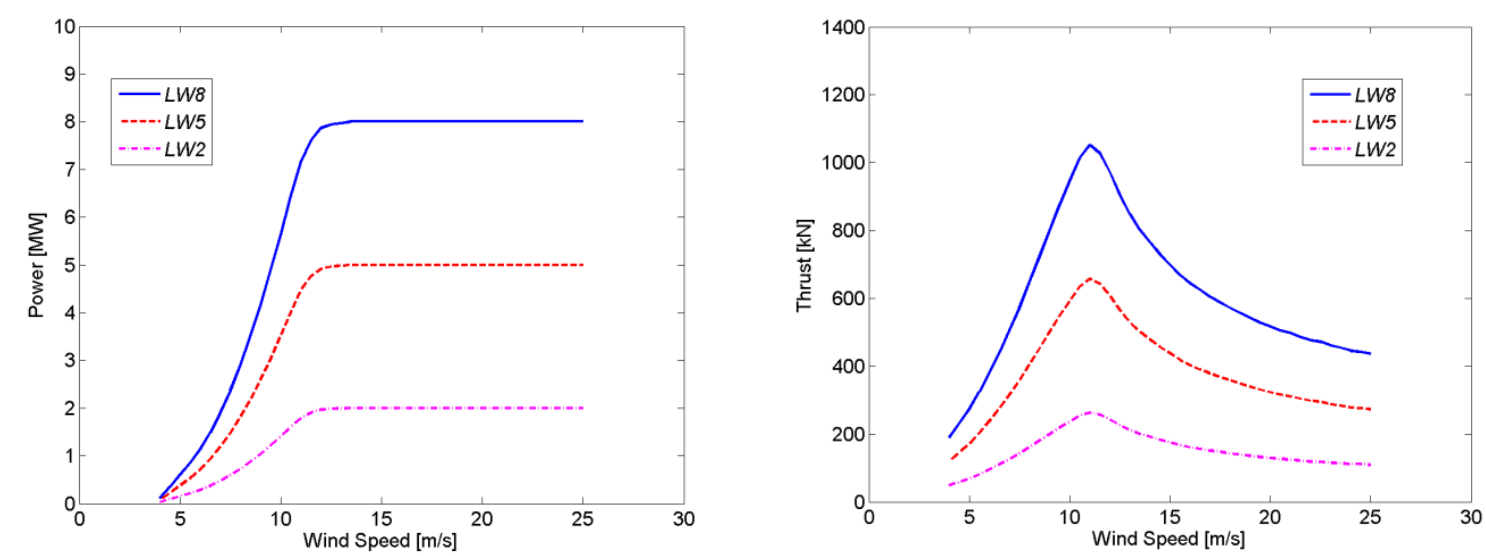

Figure 1. (left) power curves and (right) thrust curves of the 3 types of WTs

\section{Cost model}

To consider economic indicators in the optimization objective, such as LPC or COE, we need to model the cost of offshore WFs as accurately as possible. A lot of studies (e.g., [42-45]) have been done to investigate the general trend and reduction potential for cost of offshore wind power. They typically gave no analytical formulas for the different cost components, and consequently their results are difficult to use in detailed WF design optimization studies. The existing cost calculation tools, such as the LCOE model [46] developed by Megavind, Denmark's national partnership for wind energy, often require input of price for different components. While very relevant for industrial practitioners and policy makers, this kind of cost models are not adequate for researchers (mostly from the academia) to apply in WF design optimization studies. 
Consequently, in the published studies of WF design optimization, researchers often rely on some generic cost models. For example, a fixed amount of cost per MW for the capital cost and a fixed proportion of the total cost for the O\&M cost are typically assumed, as described in [47] (on page 531) and used in several studies, e.g., $[8,11]$. Alternatively, an simplified cost model depending only on the number of WTs $(N)$ is used, e.g., Cost $=N\left(\frac{2}{3}+\frac{1}{3} e^{-0.00174 N^{2}}\right)$, which was first proposed in the seminal work by Mosetti et al. in 1994 [5] and adopted in a lot of latter studies, e.g., [48-50] (a more complete list can be found in [6]). A response surface-based WF cost model proposed by Zhang et al. [51] was also adopted by Chowdhury et al. [23,31]. For non-uniform WFs, this cost model depends only on the rated power and the number of WTs for each type of WT [23].

More detailed cost models have also been proposed. For example, Dicorato et al. [52] developed a model to evaluate the total investment cost of an offshore WF, considering costs of turbine (including foundation), electric system (including collection, integration and transmission systems), grid interface and project development. Shafiee et al. [53] proposed a parametric whole life cost model for offshore WFs, based on a combined multivariate regression/neural network approach. Although not applied yet, these advanced cost model can be quite useful for a complete offshore WF design optimization that involves other design factors such as electrical system.

Moreover, it is well known that the cost of offshore WFs is quite site specific and depends on a lot of factors, such as the water depth, the distance to shore, and the labor cost. This has been clearly demonstrated in a recent study by Morthorst el al. [54], in which large variations of average investment cost per MW of offshore WFs were found from country to county, and from WF to WF, based on a comprehensive analysis of data from 45 large European offshore WFs. They also found that the average investment cost per MW has in general been increased for offshore WFs, and attributed this phenomenon mainly to: exploring deeper waters further from the shore, supplying bottlenecks and higher commodity prices. Similar observations and explanations were also given by Gonzalez-Rodriguez in a recent review [55]. This review analyzed the data available in the literature related to the cost of offshore WFs, including costs of WTs, foundations, electrical infrastructure, design and project management and O\&M. Based on the analysis, regression curves are fitted to represent the costs of different components/factors as functions of the WF total capacity. While these curves can be useful when assessing the cost of a given offshore WF, they are not adequate for applications in an overall design optimization, since they are only functions of the total capacity and thus don't change with the specific designs.

In view of the current status of cost modelling for offshore WFs and considering the fact that only the number, type and location of WTs are explicitly treated as design variables in this study, we choose to use a cost model that is a function of the total capacity and capacity factor of the WF, 
together with the composition of different types of WTs. This model is based on the published results in $[56,57]$ and described in the following sub-sections.

\subsection{Capital cost}

The capital cost model is adopted from the INNWIND cost model [56]. It is divided into turbine cost and balance of plant (BoP) cost, and based on a reference offshore WF composed of 5MW reference WTs. The BoP cost includes the cost of foundation system, offshore electrical system, offshore transportation and installation. Following the reference case in [56], the turbine cost is assumed to be $T C_{r e f}=7.5 \mathrm{M} €$ and the BoP cost is $B C_{r e f}=10 \mathrm{M} €$. Then the mean cost for different turbine sizes is scaled from the reference 5MW WT following the upscaling methodology described in [56].

For a scaled WT with rated power $P_{\text {scaled }}$, its size scale-up factor to the reference WT is defined as $s=\sqrt{P_{\text {scaled }} / P_{\text {ref }}}$. Assuming the upscaling exponents are $\lambda_{T C}$ for the turbine cost and $\lambda_{B C}$ for the BoP cost, we can calculate the mean capital cost of this scaled WT as:

$$
C A P E X_{\text {mean }}=\frac{T C_{\text {ref }} \cdot\left(\frac{P_{\text {scaled }}}{P_{\text {ref }}}\right)^{\frac{\lambda_{T C}}{2}}+B C_{\text {ref }} \cdot\left(\frac{P_{\text {scaled }}}{P_{\text {ref }}}\right)^{\frac{\lambda_{B C}}{2}}}{P_{\text {scaled }}}
$$

Note that $P_{r e f}=5 \mathrm{MW}$ is used in this study as in [56].

Different cost scenarios can be modelled by different values of the upscaling exponents. If real data on the costs of different sized WTs and WFs are available, they can be used to derive the upscaling exponents based on parameter fitting.

However, as we have no access to real price data, we can't obtain such derived exponents. Besides, as a research work, this study mainly aims to explore the potential benefits of using non-uniform design under different cost scenarios and develop an efficient method to solve its optimization problem.

To cover different cost comparison scenarios, we consider four cost scenarios with different upscaling exponents. Scenario 1 assumes a constant capital cost per MW for all WT sizes $\left(\lambda_{T C}=2\right.$, $\left.\lambda_{B C}=2\right)$. Scenario 2 uses the exponents $\left(\lambda_{T C}=2.42, \lambda_{B C}=1.50\right)$ from the innovative design in [56], which assumes the capital cost per MW decreasing with WT size. Scenario 3 is a scenario $\left(\lambda_{T C}=\right.$ 2.77, $\lambda_{B C}=1.75$ ) that assumes the capital cost per MW increasing with WT size. Possible reasons behind this scenario could be higher technology maturity and existing large scale manufacture capacities for the smaller size WTs, for example, the popular 2MW WTs. Scenario 4 is the classical upscaling scenario $\left(\lambda_{T C}=3, \lambda_{B C}=2\right)$ which results in a much higher cost per MW for larger WTs. The resulting curves for capital cost are shown in Figure 2 for these 4 scenarios. Note that the green markers show the mean capital costs of the 3 types of WTs under different scenarios. 


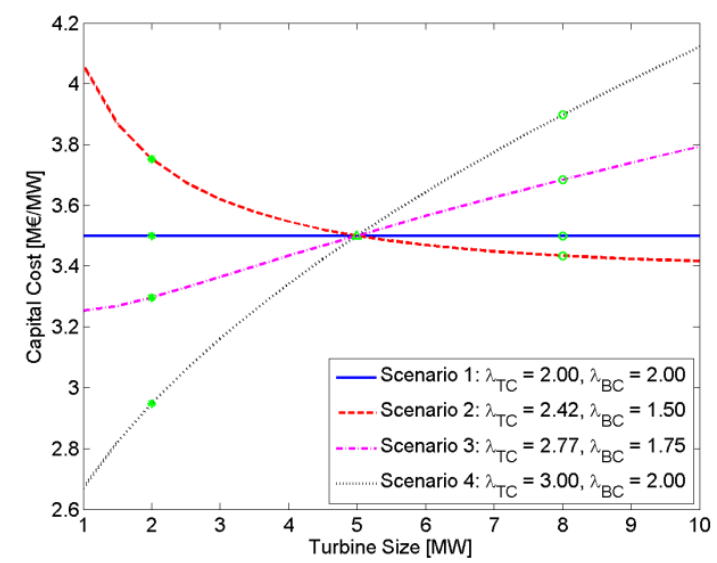

Figure 2. Capital cost per MW for different WT sizes under 4 scenarios

For the 3 types of WTs used in this study, their capital costs under different scenarios are presented in Table 2.

Table 2. Capital cost per MW for the 3 types of WTs

\begin{tabular}{cccccc}
\hline \multirow{2}{*}{ Scenario } & \multirow{2}{*}{$\lambda_{T C}[-]$} & \multirow{2}{*}{$\lambda_{B C}[-]$} & \multicolumn{3}{c}{ Capital cost [M€/MW] } \\
& & & LW2 & LW5 & LW8 \\
\hline 1 & 2.00 & 2.00 & 3.50 & 3.50 & 3.50 \\
2 & 2.42 & 1.50 & 3.75 & 3.50 & 3.43 \\
3 & 2.77 & 1.75 & 3.30 & 3.50 & 3.68 \\
4 & 3.00 & 2.00 & 2.95 & 3.50 & 3.90 \\
\hline
\end{tabular}

\subsection{Levelised cost of energy (LCOE)}

LCOE of a given offshore WF is calculated by using the model with the key performance indicators from the European Wind Industrial Initiative (EWII) [57]. Considering an offshore WF composed of a given number of the 3 types of WTs, we can calculate its total capital cost CAPEX total based on the mean capital cost of each turbine type. When the offshore WF design and the wind distribution are given, the expected total power output $P_{\text {total }}$ can be computed by considering the wake effects using a wake model, such as the Jensen wake model $[58,59]$. Note that the procedure of computing $P_{\text {total }}$ is described in section 4.2. Then the annual energy production of this WF is given by:

$$
A E P=P_{\text {total }} \cdot 8760 \cdot \eta
$$

where 8760 is the total number of hours in one year and $\eta$ is the availability factor. We assume $\eta=$ 1.0 in this study, mainly because there is no model available to account the possible dependency of available factor on different WF designs and a fixed value of $\eta$ will affect the LCOE (as calculated in Eq. (5)) of different WF designs in the same manner thus has no influence on the optimization results. If the offshore WF total capacity is denoted as Capacity, its capacity factor can then be written as: 


$$
C F=\frac{P_{\text {total }}}{\text { Capacity }}
$$

Denoting the annualized O\&M cost as OPEX annual, we can use the model in [57] to get:

$$
\text { OPEX } X_{\text {annual }}=O P E X_{\text {ref }} \cdot \text { Capacity } \cdot\left[1+0.5 \cdot\left(C F-C F_{\text {ref }}\right)\right]
$$

where $O P E X_{\text {ref }}=106 € / \mathrm{kW} /$ year is the baseline mean O\&M cost for offshore wind and $C F_{\text {ref }}=$ 0.4 is the baseline capacity factor for the reference case. Finally, LCOE of this WF can be computed as:

$$
L C O E=\frac{C A P E X_{\text {total }} \cdot C R F+O P E X_{\text {annual }}}{A E P}
$$

where $C A P E X_{\text {total }}$ is the total capital cost consists of costs relating to each type of WTs, and $C R F$ is the capital recovery factor depending on the discount rate $d$ and the projected lifetime $N$ (year). The formulas to calculate $C A P E X_{\text {total }}$ and $C R F$ are as follows:

$$
\begin{gathered}
\text { CAPEX }_{\text {total }}=\sum_{j=1}^{N_{\text {types }}} C_{\text {APEX }}^{j} \cdot \text { Capacity }^{j}, \\
C R F=\frac{d}{\left[1-(1+d)^{-N}\right]}
\end{gathered}
$$

where CAPEX $_{\text {mean }}^{j}$ and Capacity $^{j}$ denote the mean capital cost per MW and the capacity of the $j$ th type of WTs, respectively; $N_{\text {types }}$ represents the number of involved WT types. In this study, the values recommended in [57], i.e., $d=5.39 \%$ and $N=25$ are used for calculating $C R F$.

\section{Design optimization problem}

\subsection{Problem formulation}

Considering the design of a non-uniform offshore WF that can choose from $N_{\text {types }}$ types of WTs, we can specify each WT by its location, type and related hub height. Thus, the design of this offshore WF can be denoted as $\boldsymbol{L}=\left[x_{i}, y_{i}, T_{i}, H_{i}\right]$ with $i=1,2, \ldots, N_{w t}$. Note that $T_{i}$ is an integer in the range of 1 to $N_{\text {type }}$ that denotes the type code of the $i$ th turbine. In this work, $T_{i}=1,2$ or 3 represents the type of the WT is LW2, LW5 or LW8, respectively. Although the above formulation includes the hub heights, we assume that only the default hub height is used for a given turbine type in the case study, thus $H_{i}$ could be safely removed. Anyhow, we keep the general formulation including hub heights to indicate that the methodology developed in this study can easily consider the hub height variations. Note that similar formulation of WF design was adopted in other studies, such as [23].

Noticing that $L C O E$ of a given offshore WF in Eq. (5) is essentially a function of its design, we can write the optimization problem of minimizing the offshore WF LCOE as: 


$$
\min \quad L C O E=f\left(\boldsymbol{L}, N_{w t}\right)
$$

Three types of constraints are considered. The first type is the constraint on the total capacity, which comes from the requirement of electricity demand and limitation of grid transmission capacity. It can be written as:

$$
\operatorname{Capacity}^{(L)} \leq \operatorname{Capacity}_{\left(\boldsymbol{L}, N_{w t}\right)} \leq \operatorname{Capacity}^{(U)}
$$

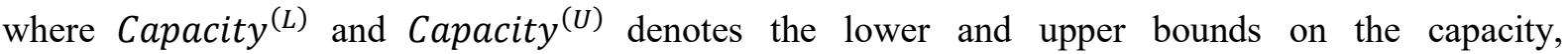
respectively.

The second type is the constraint on the feasible area, which can be specified as $S_{\text {feasible }}$ defined by the boundary and other possible forbidden zones. It is governed by:

$$
\left(x_{i}, y_{i}\right) \in S_{\text {feasible }}, \quad \text { with } i=1,2, \ldots, N_{w t} .
$$

The third type is the constraint on the proximity distance of any pair of WTs, which can be written as:

$$
\sqrt{\left(x_{i}-x_{j}\right)^{2}+\left(y_{i}-y_{j}\right)^{2}} \leq \overline{D i s t}_{\text {min }} \cdot \max \left(D_{i}, D_{j}\right), \quad \text { with } \begin{aligned}
& i=1,2, \ldots, N_{w t}-1, \\
& j=i+1, \ldots, N_{w t} .
\end{aligned}
$$

where $\overline{D i s t}_{\min }$ is the dimensionless minimal distance requirement and $D_{i}$ denotes the rotor diameter of the $i$ th WT. We use $\overline{D i s t}_{\text {min }}=5$ to assure any two WTs are at least 5 rotor diameters (the diameter of the larger WT) away from each other.

\subsection{Offshore WF modelling}

Calculating the power production accurately is essential in the context of offshore WF design optimization, since the most important function of an offshore WF is to generate power. This requires the appropriate modelling of wind, wake effects and power production.

There are multiple types of distributions that can be used to model the probability distribution of offshore wind [60]. However, it is the common practice to model wind resource with sector-wise Weibull distributions and wind rose [61]. In general the wind conditions at a given height can be described by wind speed $v$ and wind direction $\theta$, and its distribution can be modelled by a probability density function (PDF) $p d f(v, \theta)$. In our previous study [62], a joint distribution of wind speed and wind direction was proposed, which can be used to obtain PDF based on real measurement data. In this study, we use the real wind characteristics as measured at Horns Rev in Denmark, the obtained joint distribution is shown in Figure 3. 


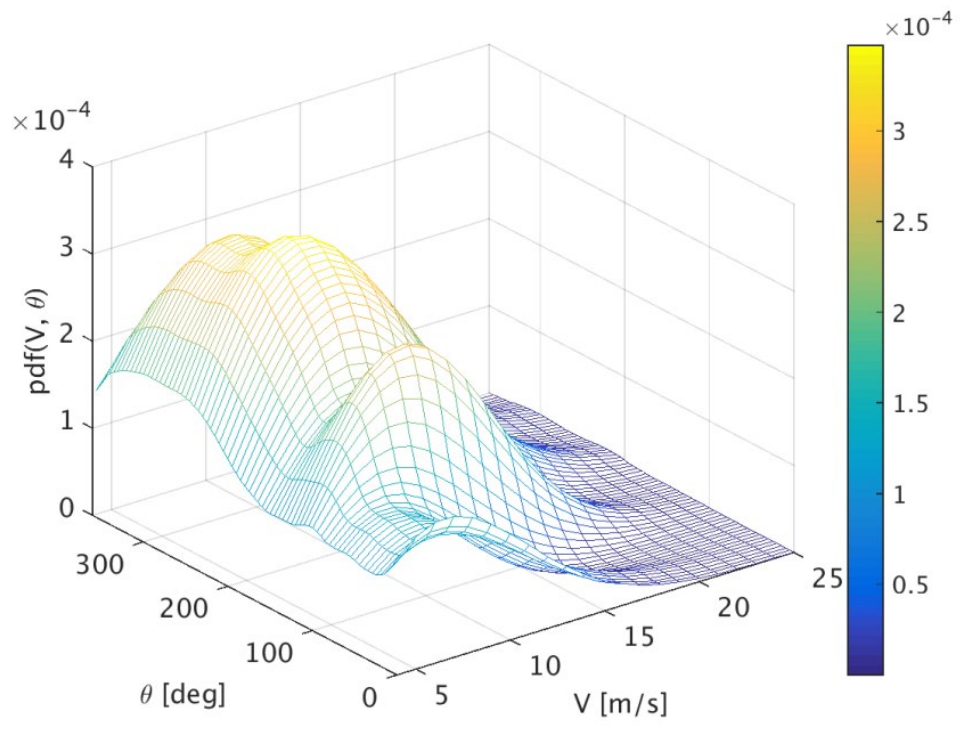

Figure 3. Joint distribution of wind speed and wind direction at Horns Rev [62]

The measured wind data might be obtained at a reference height $H_{r e f}$ and then used to predict the power production of a WT with hub height $H$, which is different for different types of WTs. It is thus necessary to first transfer the measured wind speed $v_{r e f}$ into the inflow wind speed $v$ at hub height $H$, usually using the logarithmic law:

$$
v=v^{H}\left(v_{r e f}\right)=v_{\text {ref }} \frac{\ln \left(H / z_{0}\right)}{\ln \left(H_{r e f} / z_{0}\right)}
$$

where $v_{r e f}$ denotes the wind speed at the measured height and $z_{0}$ is the surface roughness length [47]. Note that the Horns Rev measurement was done at $70 \mathrm{~m}$ height and we assume $z_{0}=0.0001 \mathrm{~m}$.

Wake effect arises when an upwind WT extracts energy from the wind and forms a wake that impacts the downwind WTs. It leads to reduced wind speed and increased turbulence, and thus is crucial for offshore WF modelling. Due to the nature of optimization problem, engineering wake models are commonly used because of their low computational costs. Most of the published studies (such as $[5,8,9,11,12,14,17,27,28,34-36]$ ) on offshore WF layout optimization used the Jensen wake model [58,59], which is also called as the Park model. The effectiveness of this wake model has been confirmed in several studies when comparing with measurement data, such as in $[63,64]$. A comparison with large eddy simulation for a real 48-turbine offshore WF (Lillegrund in Sweden) also validated its capability of modelling wake effects for WTs with different hub-heights [36]. Considering its wide popularity, simplicity and low computational cost, we use the Jensen wake model in this study. 
For a given freestream inflow condition $(v, \theta)$, the wake effects between WTs can be first calculated using the Jensen wake model and then the effective wind speed at each WT $\left(\bar{v}_{i}\right.$, i.e., the effective wind speed the $i$ th WT experienced) can be evaluated. The power generated by each WT under this inflow condition can then be calculated based on the manufacturer provided power curve $P=P(v)$. Combined with the PDF of wind condition, the expected total power produced by the WF can be written as

$$
P_{\text {tot }}=\sum_{i=1}^{N_{w t}} \iint P\left(\bar{v}_{i}\right) \times p d f(v, \theta) d v d \theta
$$

The detailed procedure for calculating power production can be found in [14].

\subsection{Baseline designs}

Among operational offshore WFs, the majority are composed of identical types of WTs arranged in symmetrical and grid-like layouts [17]. As reference cases, we propose three baseline designs with the same total capacity (160MW) but each composed of a different type of WTs. The first one is composed of 80 LW2 WTs, organized in a grid like layout with 7 by 7 rotor diameters spacing. The offshore WF occupies a rectangle area with size $5166 \mathrm{~m}$ by $4018 \mathrm{~m}$. Note that its layout and spacing between WTs are specified in the similar manner as the Horns Rev 1 WF [14]. The second and third designs are constructed in similar manner with 32 LW5 and 20 LW8 WTs, occupying the same area as the first one. The three designs are shown in Figure 4, with their characteristics presented in the titles. The efficiency values in these titles are WF efficiencies, which are calculated as the real expected total power, i.e., the power production computed using Eq. (12), divided by the ideal expected total power, i.e., the power production if there are wake effects.

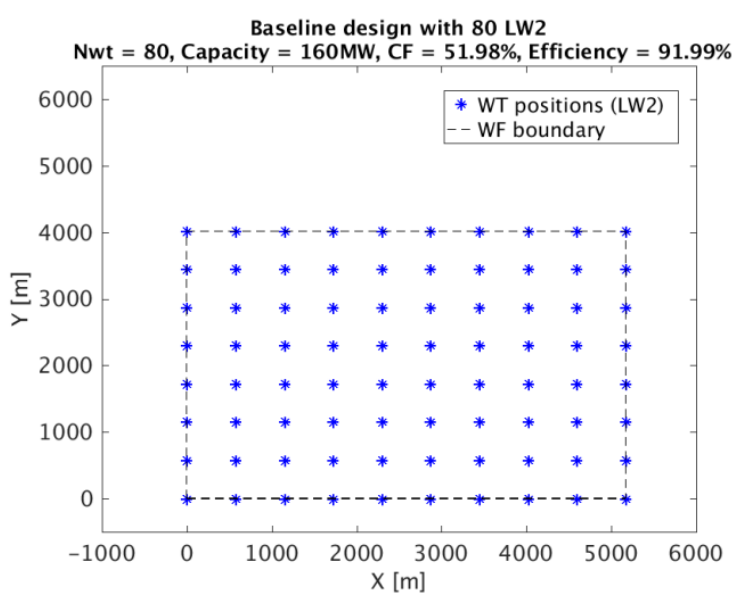

(a) Baseline1

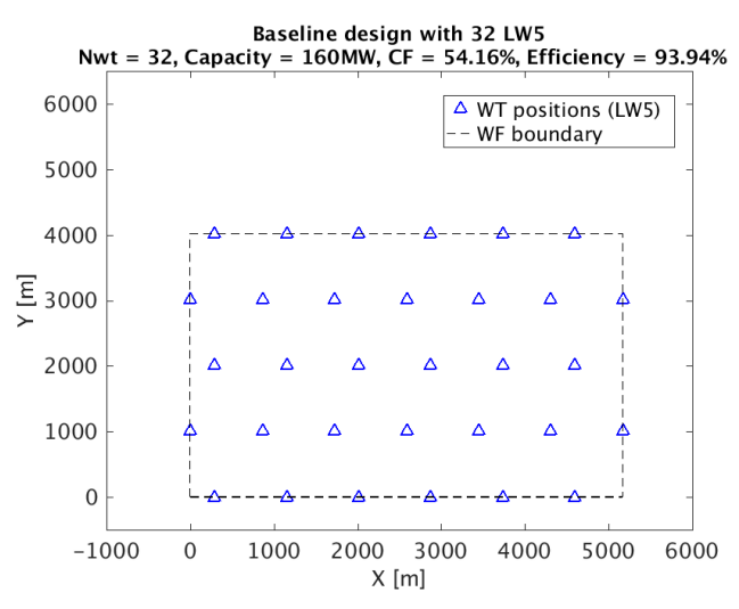

(b) Baseline2 


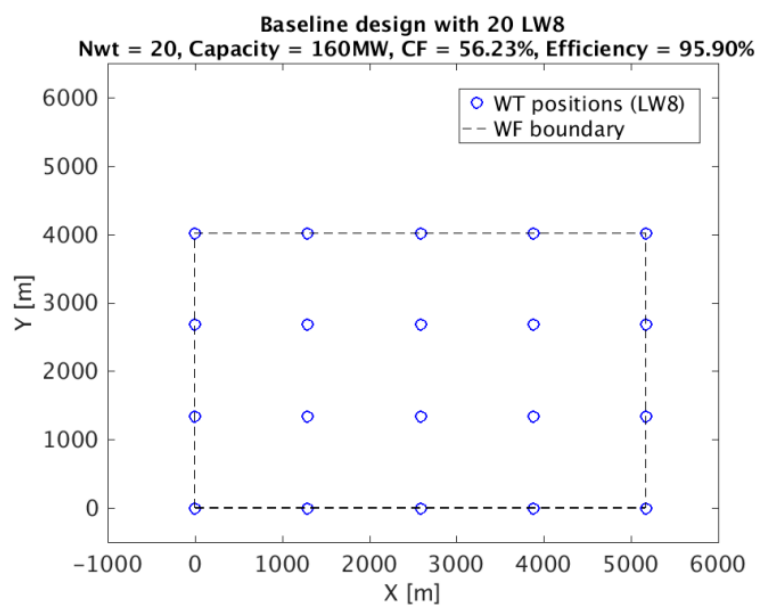

(3) Baseline3

Figure 4. Three baseline designs composed of single type of WTs

Table 3 shows the LCOE of these baseline designs under the 4 different cost scenarios. We can see that their relative ranking in terms of LCOE varies with cost scenario.

Table 3. LCOE of baseline designs

\begin{tabular}{cccc}
\hline \multirow{2}{*}{ Scenario } & & LCOE [€/MWh] & \\
& Baseline1 (LW2) & Baseline2 (LW5) & Baseline3 (LW8) \\
\hline 1 & 81.36 & 78.33 & 75.66 \\
2 & 85.45 & 78.33 & 74.67 \\
3 & 78.07 & 78.33 & 78.41 \\
4 & 72.43 & 78.33 & 81.61 \\
\hline
\end{tabular}

When the capital cost per MW is constant or decreases with increasing WT size, as shown in Scenario 1 and 2, the baseline designs with larger WTs always outperform. This can be explained by comparison of capacity factors as shown in Figure 4. For the design with larger WTs: the hub heights are higher, thus the wind resource is better; the number of WTs are smaller and the spacing is larger in terms of rotor diameters, thus the wake effect is weaker (also suggested by the higher WF efficiency). Therefore the capacity factor ranks as: Baseline $3>$ Baseline $2>$ Baseline1. Thus, the higher AEP combined with the same or cheaper capital cost makes WFs composed of a larger size turbines more competitive in LCOE.

On the other hand, when the capital cost per MW increases with WT size (in Scenarios 3 and 4), the baseline designs with smaller WTs are better. Comparing Baseline1 and Baseline3 in Scenario 3, we can see that the difference in LCOE between these two designs is quite small $(0.44 \%)$, while the 
difference in capital cost per MW between LW2 and LW8 is much larger (11.52\%), which is mainly due to the higher capacity factor achieved by using larger WTs (LW8).

In general, the above comparisons show that, depending on the different cost scenarios, different types of WTs have their different competitive advantages, which clearly suggests that it will be beneficial to investigate the non-uniform design options.

\section{Optimization algorithm}

In our previous studies, the RS algorithm has been developed for uniform offshore WF layout optimization [14]. In this section, we present an extended RS that can solve the non-uniform offshore WF design optimization problems involving multiple types and unfixed number of WTs.

This algorithm is a single solution search method. At each step, a new feasible solution is generated by repeating $N_{\text {repeat }}$ times of one of the following actions to the current solution: adding, removing, or changing one WT randomly. Note that changing one WT means changing its type and/or location (and/or hub height if hub height variation is considered). The choice among these 3 actions is made based on specified probabilities. This new solution is then compared with the current solution. If the new solution is better, it is used to replace the current solution. This step is iteratively repeated until a stop condition is met. Normally, the stop condition can be set as a given maximal number of steps (or WF evaluations). The procedure of this algorithm is shown as flow chart in Figure 5.

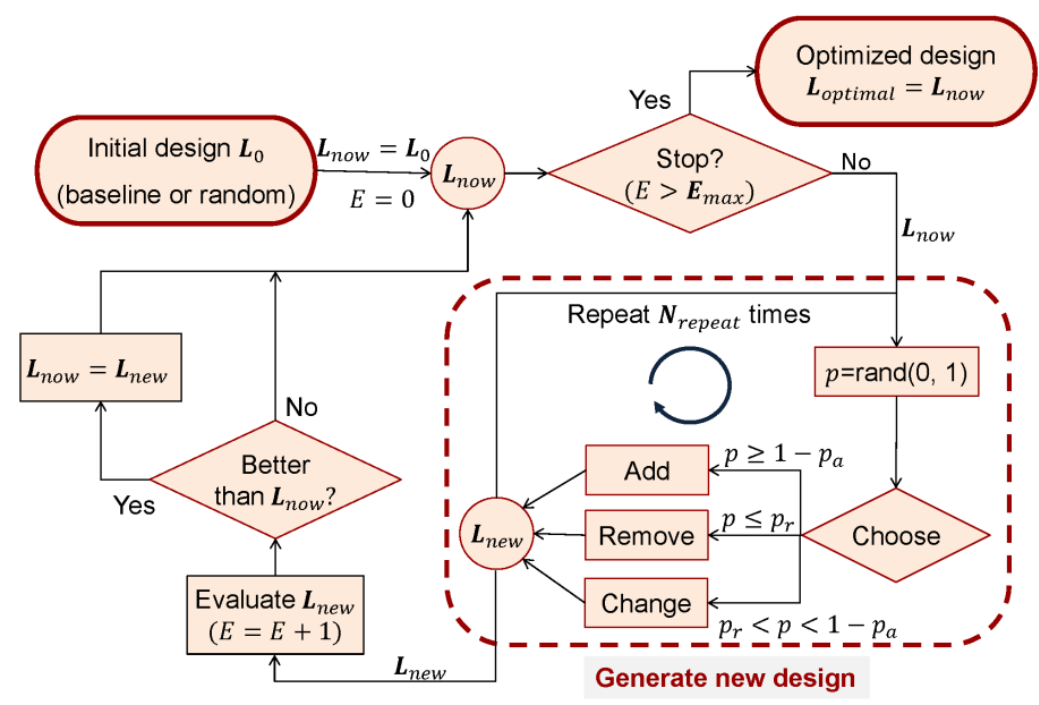

Figure 5. Flow chart of the extended random search algorithm

Note that at each step, any action done to the offshore WF design, i.e., adding, removing or changing one WT, is made to produce a changed design that satisfies all the constraints. By doing this, 
we ensure the generated new design at each step is always feasible. Assuming the maximal number of evaluations is $E_{\max }$, we can see there are only four parameters to tune for this algorithm: $N_{\text {repeat }}$, $E_{\text {max }}, p_{a}$ and $p_{r}$. Thus, this algorithm is quite simple, intuitive and easy to implement. Among the four parameters, $N_{\text {repeat }}$ is used to control how different the generated new design is from the current best design, thus determines the trade-off between global exploration and local exploitation; $E_{\text {max }}$ serves as the stop condition; $p_{a}$ and $p_{r}$ specify the portions of adding and removing actions taken when changing a design, respectively. For different problems, these parameters should be tuned based on some numerical experiments.

\section{Optimization results}

The extended RS algorithm described above, as well as other widely used algorithms in WF design optimization (e.g., GA [5,11,48] and PSO [23,31,49]), belongs to the family of metaheuristics [65]. Metaheuristics are particularly suitable for highly constrained and high dimensional nonlinear optimization problems, such as the WF design optimization [27]. For such kind of complex optimization problems, the global optimum, as well as how to prove whether a solution is the global optimum, is usually unknown. Thus, one usually has to live with a local optimum. Nevertheless, a well selected and tuned metaheuristic can achieve a good balance between diversification (i.e., global exploration) and intensification (i.e., local exploitation), thus obtain a good solution [66].

However, due to the randomness involved in the optimization process, metaheuristics usually have non-deterministic nature, which means different runs of a metaheuristic optimization algorithm typically yield different results [65]. This also means that the quality of the initial solutions has profound influence on the final optimization results.

For a metaheuristic to perform well, the diversity of the initial solutions needs to be ensured to enable the algorithm to search the design space more thoroughly [66]. For population based algorithms, such as GA, this means the initial population needs to be generated covering broadly the feasible design space [67]. Also the quality of solutions in the initial population could impact the quality of the final solution found by the algorithm. Among studies in WF design optimization, Saavedra-Moreno et al. [68] tackled this problem by seeding evolutionary algorithm with initial solutions obtained by a greedy heuristic algorithm, and applied this method in WF layout optimization.

For single solution search metaheuristics (also called as trajectory methods), such as RS, the diversity of initial solutions can be achieved by applying multi-start strategies [69], i.e., running the metaheuristic with multiple initial solutions that generated by a strategical construction process and using the best solution found as the final optimized solution. 
We implement the multi-start strategy in this study. For each of the baseline designs (denoted as B1, B2 and B3), we can generate 5 randomized designs by taking those actions (adding, removing or changing) certain times to the corresponding baseline design. These randomized designs are denoted as RB1s, RB2s and RB3s. To compare with uniform WF design, we also consider the cases with single type of WTs (the same turbine type as in the baseline design it starts from) in a given design. For each cost scenario, two groups of optimization works are done: using 1 type of WTs and using 3 types of WTs. For each group, 10 optimization runs are carried out starting from each of the baseline designs (5 from the baseline design and 5 from the randomized baseline designs).

To better understand the influence of initial solutions, optimization runs starting from pure random designs (denoted as PRs) are also implemented for the non-uniform design scenarios, i.e., using 3 types of WTs. These pure random designs are generated by repeating the adding action described in the last section to an empty WF until the total capacity reaches the feasible range $\left(\left[\right.\right.$ Capacity $^{(L)}$, Capacity $\left.\left.^{(L)}\right]\right)$. Two of initial designs generated in such way are shown in Figure 6.
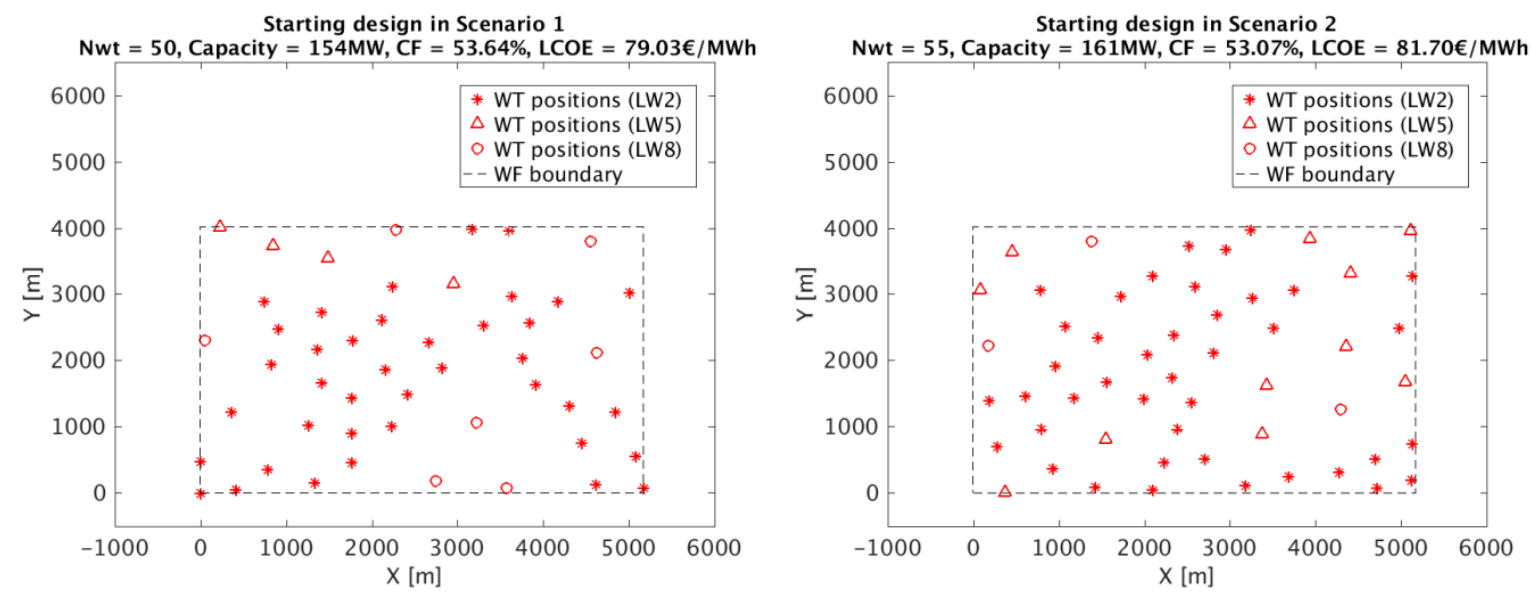

Figure 6. Two pure random designs as starting solutions for: (left) Scenario 1, (right) Scenario 2

Note that the constraints on capacity are set as Capacity $^{(L)}=152 \mathrm{MW}$ and Capacity $^{(U)}=168 \mathrm{MW}$ and the parameters used in the extended RS algorithm (selected based on initial numerical experiments) are as follows: $N_{\text {repeat }}=9, E_{\max }=10000, p_{a}=0.2$ and $p_{r}=0.6$.

The statistics of the LCOE of the obtained designs in multiple runs are summarized in Table 4, with the best results in a particular group under each scenario marked in bold for both uniform and nonuniform design options, and the overall best designs under each scenario underlined and chosen as the final optimized designs.

Table 4. LCOE of the obtained designs

\begin{tabular}{llrl}
\hline & LCOE $[€ / \mathrm{MWh}]$ & \\
Scenario 1 & Scenario 2 & Scenario 3 & Scenarios 4
\end{tabular}




\begin{tabular}{clcccccccc} 
Best Baseline & & \multicolumn{2}{c}{$75.66(\mathrm{~B} 3)$} & \multicolumn{2}{c}{$74.67(\mathrm{~B} 3)$} & \multicolumn{2}{c}{78.07 (B1) } & \multicolumn{2}{c}{72.43 (B1) } \\
\hline & & 1 type & 3 types & 1 type & 3 types & 1 type & 3 types & 1 type & 3 types \\
\hline \multirow{2}{*}{ From B1/RB1s } & min & 81.00 & 80.58 & 85.07 & 74.38 & 77.72 & 77.72 & $\mathbf{7 2 . 0 8}$ & $\mathbf{7 2 . 1 1}$ \\
(10 runs) & $\operatorname{mean}$ & 81.02 & 80.91 & 85.10 & 75.96 & 77.75 & 77.74 & 72.12 & 72.14 \\
& $\max$ & 81.06 & 81.03 & 85.21 & 85.07 & 77.79 & 77.78 & 72.14 & 72.19 \\
\hline \multirow{2}{*}{ From B2/RB2s } & $\min$ & 77.63 & 75.74 & 77.61 & $\mathbf{7 4 . 3 8}$ & $\mathbf{7 7 . 6 1}$ & $\mathbf{7 7 . 4 7}$ & 77.64 & 74.02 \\
(10 runs) & $\operatorname{mean}$ & 77.71 & 77.36 & 77.69 & 74.85 & 77.69 & 77.61 & 77.70 & 75.52 \\
& $\max$ & 77.77 & 79.01 & 77.74 & 75.39 & 77.79 & 77.72 & 77.75 & 76.58 \\
\hline \multirow{2}{*}{ From B3/RB3s } & $\min$ & $\mathbf{7 5 . 3 9}$ & $\mathbf{7 5 . 4 2}$ & $\mathbf{7 4 . 4 0}$ & 74.47 & 78.14 & 77.51 & 81.36 & 75.81 \\
(10 runs) & $\operatorname{mean}$ & $\mathbf{7 5 . 4 5}$ & 76.05 & 74.47 & 74.66 & 78.20 & 77.91 & 81.41 & 76.22 \\
& $\max$ & 75.49 & 77.31 & 74.51 & 75.31 & 78.25 & 78.29 & 81.43 & 76.55 \\
\hline \multirow{2}{*}{ From PRs } & $\min$ & $/$ & 76.47 & $/$ & 74.48 & $/$ & 77.53 & $/$ & 74.20 \\
(10 runs) & $\operatorname{mean}$ & $/$ & 77.14 & $/$ & 74.66 & $/$ & 77.69 & $/$ & 75.08 \\
& $\max$ & $/$ & 77.88 & $/$ & 74.99 & $/$ & 77.81 & $/$ & 76.18 \\
\hline
\end{tabular}

Further examining the results in Table 4, we can clearly see the importance of using multi-start strategy with different initial designs. Especially for the uniform design cases, i.e., the 1 turbine type cases, the quality of the initial designs largely determines the quality of the optimized designs. Note, however, that the allowed type of turbine is LW2 for runs from B1/RB1s, LW5 for B2/RB2s and LW8 for B3/RB3s, which means there are essentially 3 different uniform design optimization problems we are solving here, each using a different type of turbine. Thus, the large differences between the results from different groups of starting solutions (i.e., B1/RB1s, B2/RB2s and B3/RB3s) in the uniform design cases should be mainly attributed to the fact that different types of turbine are used.

For the runs from the same group of starting designs, e.g., B1/RB1s, the LCOE ranges of the optimized designs obtained in different runs are also smaller for the ' 1 turbine type' cases than the ' 3 turbine types' cases. This can be explained by the fact that using uniform WF design is essentially narrowing the search space in the design variable space, thus limiting its global exploration ability. In contrary, the non-uniform design option, i.e., the option with 3 types of turbines to choose from, usually exhibits larger diversity in the obtained optimization results.

Nevertheless, under all cost scenarios, the best solutions found with the non-uniform design option are either better or very close to the best solutions obtained with the uniform design option. Considering the design space the algorithm searches is larger with the non-uniform design option, one could expect the possibility of find even better design than the current best design is also likely to be higher for the non-uniform design option, if the number of runs and the allowed evaluations for each run are increased.

The best WF designs under 4 cost scenarios, both for uniform option (i.e., with 1 turbine type to choose) and non-uniform option (i.e., with 3 turbine types to choose), are shown in Figures 7-10. 

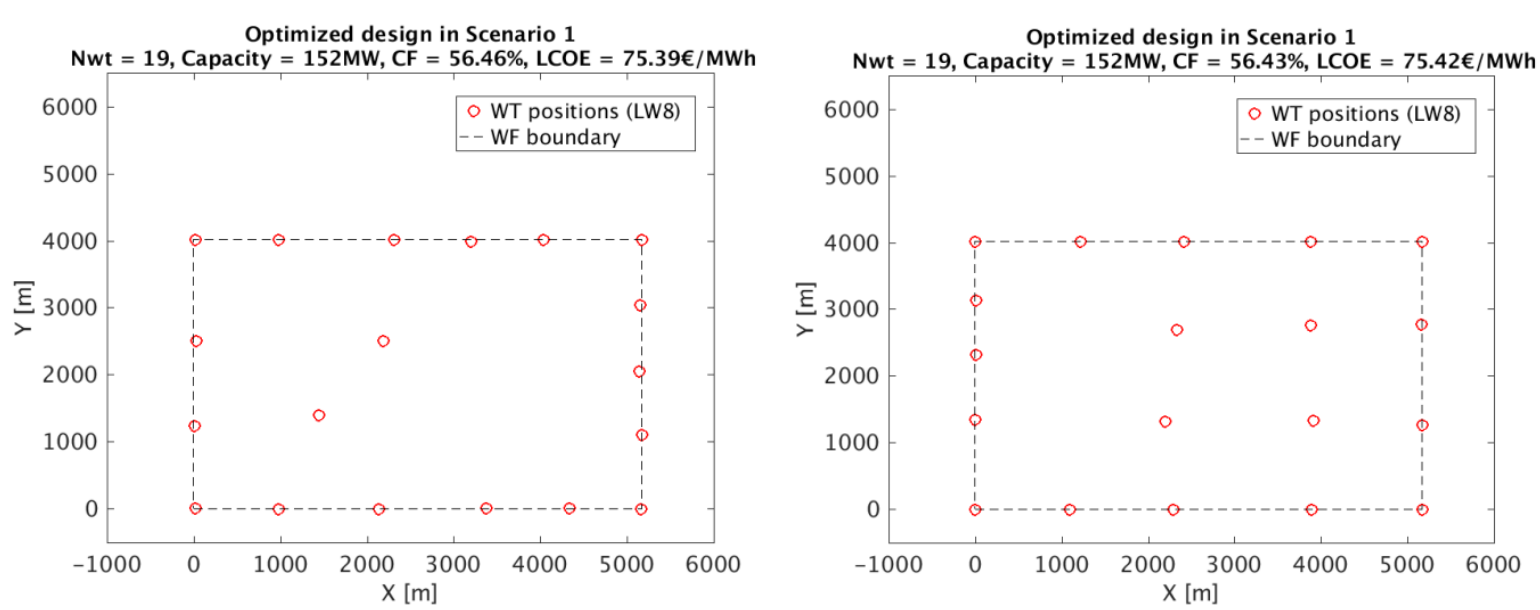

Figure 7. Optimized design in Scenario 1: (left) with 1 turbine type, (right) with 3 turbine types
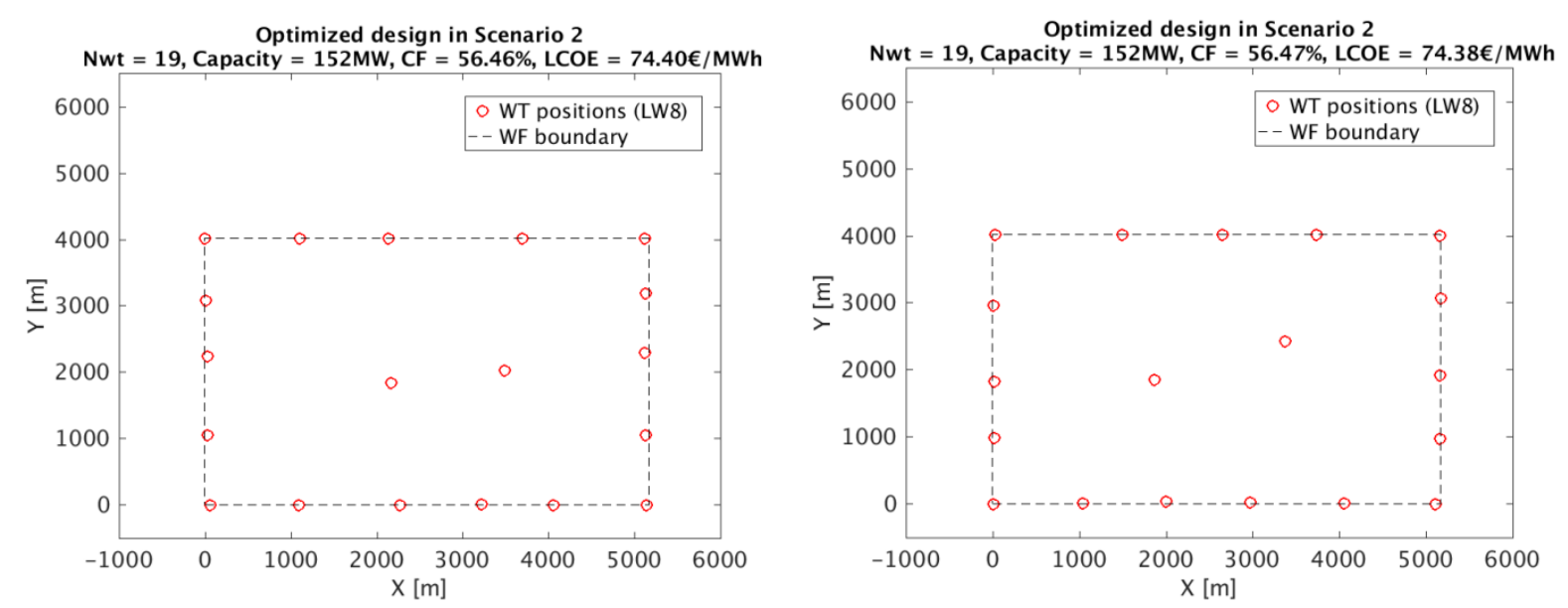

Figure 8. Optimized design in Scenario 2: (left) with 1 turbine type, (right) with 3 turbine types
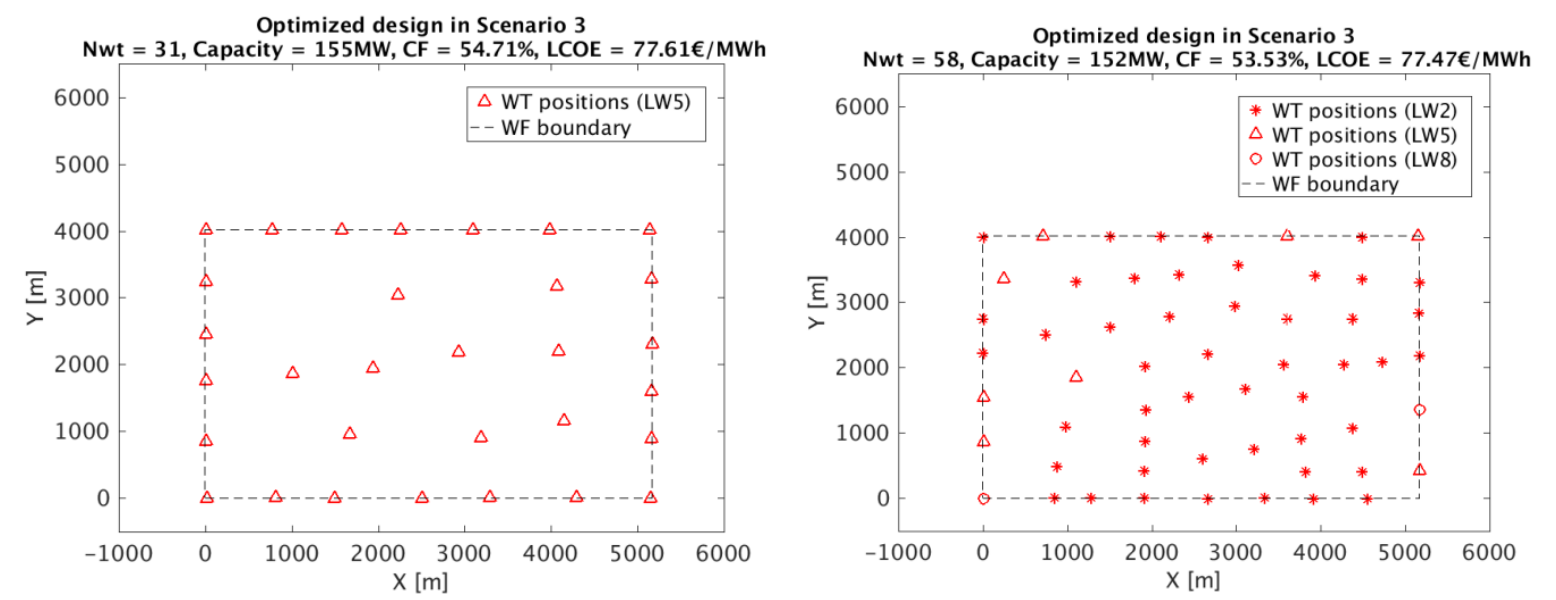

Figure 9. Optimized design in Scenario 3: (left) with 1 turbine type, (right) with 3 turbine types 

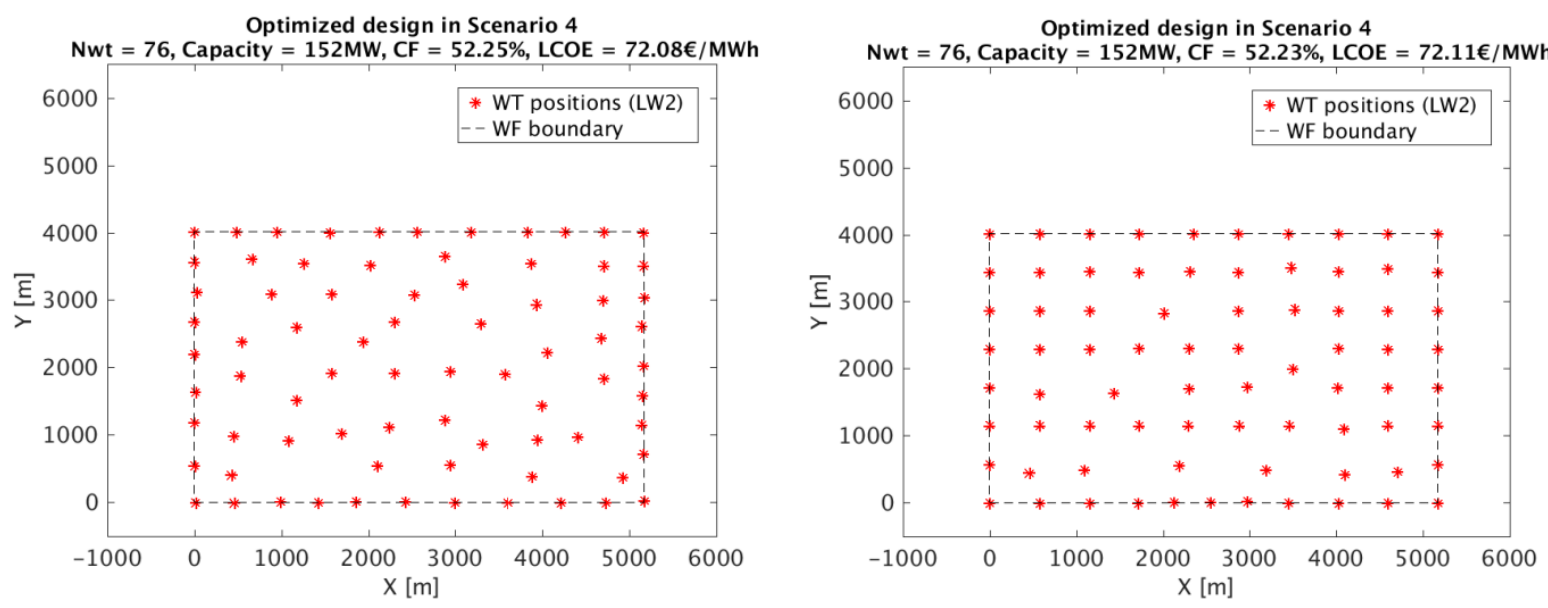

Figure 10. Optimized design in Scenario 4: (left) with 1 turbine type, (right) with 3 turbine types

We can see from these figures, the optimized designs all have the lowest allowed capacity $(152 \mathrm{MW})$. This can be explained by the fact that an offshore WF with a lower capacity typically has fewer WTs and thus less wake effects, which makes the AEP decrease sub-linearly with capacity, while the capital cost decreases linearly with the capacity in the cost model as shown in Eq. (5). When the capital cost per MW of large WTs is the same (Scenario 1) as or lower (Scenario 2) than that of small size WTs, the optimized design consists of a single type of WTs (the largest one). When the capital cost per MW of small size WTs is significant lower (Scenario 4), the optimized design also consists of a single type of WTs (the smallest one). When the capital cost per MW of small size WTs is lower but not significantly (Scenario 3), the optimized design contains 3 types of WTs.

When we compare the optimized designs with only one type of WTs to choose and those with 3 types to choose, it is seen that they generally obtain similar results. Under Scenarios 1, 2 and 4, the final obtained designs with non-uniform design option are also composed of single type of WTs, even there are 3 different types of WTs can be selected from. While under Scenario 3, the latter option obtains a different design that is composed of 3 types of WTs and also has a much lower LCOE.

Comparing the results of runs from pure random starting solutions (PRs) with those from other groups of starting solutions (baseline or randomized baseline designs), one can see that the performance of runs from PRs is in general moderate. Using pure random starting solutions typically yields designs that are worse than those obtained from good group of baseline or randomized baseline designs (e.g, B1/RB1s in Scenario 4) but better than those from bad group of baseline or randomized baseline designs (e.g. B3/RB3s in Scenario 4). This can be seen from the convergence history comparison between 3 runs in Scenario 1, as shown in Figure 11. 


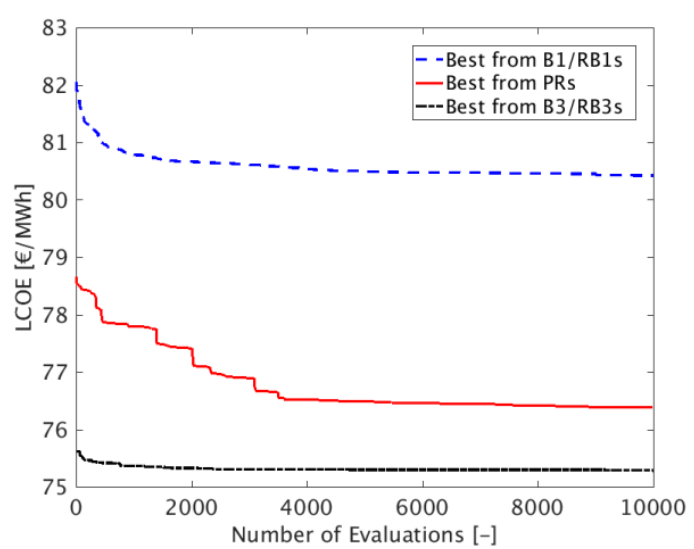

Figure 11. Convergence histories of 3 runs in Scenario 1 (the best runs from B1/RB1s, PRs and $\mathrm{B} 3 / \mathrm{RB} 3 \mathrm{~s})$

Anyway, results in this case study show that the final best designs are from the runs using baseline or randomized baseline designs. Especially for Scenarios 1, 2 and 4, in which the final best designs are composed of single type of WTs, the designs obtained using PRs are not satisfactory. Further examining the obtained designs, it is found that runs from PRs under Scenarios 1 and 4 fail to find final solutions that are composed of single type of WTs. This demonstrates the importance of maintaining diversity of initial solutions for multi-start strategy. For design optimization of nonuniform WFs, it is always advised to include the initial solutions that are uniform, i.e., designs with single type of WTs.

This recommendation can also be generalized to any mixed integer-discrete-continuous nonlinear optimization problem. When a meta-heuristic algorithm is applied to solve this kind of problems, it is usually a good idea to include some uniform initial solutions, i.e., solutions with identical value for the discrete design variables, as these uniform solutions are special 'corner cases' in the design space that could be hard for the algorithm to reach in the search process.

\section{Comparison with the mixed-discrete PSO}

To further demonstrate its effectiveness, the performance of the extended RS is compared with the mixed-discrete PSO for a non-uniform WF design optimization test case. The mixed-discrete PSO is a modified version of the conventional PSO that can adequately address constraints while dealing with mixed-discrete variables. This algorithm was developed by Chowdhury et al. [24] and has been applied in design optimization of WFs considering simultaneously the locations and types of turbines $[23,24]$.

For a general mixed-discrete single objective constrained minimization problem with $m$ discrete variables and a total of $n$ design variables, the design variable vector can be denoted as $\boldsymbol{X}=$ 
$\left[x_{1}, x_{2}, \ldots, x_{m}, x_{m+1}, \ldots, x_{n}\right]$. Note that in the context of design optimization of non-uniform WFs, the discrete variables are the types of turbines and the continuous variables are the $x$ and $y$ coordinates of turbine locations, thus we have $m=N_{w t}$ and $n=3 N_{w t}$.

Mixed-discrete PSO is a population-based meta-heuristic that updates a population of particles with each particle representing a solution in the design space. During the optimization process, the $i$ th particle is moved following the rules described below [24]:

$$
\begin{aligned}
& \boldsymbol{X}_{i}^{t+1}=\boldsymbol{X}_{i}^{t}+\boldsymbol{V}_{i}^{t+1}, \\
& \boldsymbol{V}_{i}^{t+1}=\alpha \boldsymbol{V}_{i}^{t}+\beta_{l} \gamma_{1}\left(\boldsymbol{P}_{i}-\boldsymbol{X}_{i}^{t}\right)+\beta_{g} \gamma_{2}\left(\boldsymbol{P}_{g}-\boldsymbol{X}_{i}^{t}\right)+\gamma_{c} \gamma_{3}\left(\boldsymbol{X}_{i}^{t}-\boldsymbol{P}_{g}\right)
\end{aligned}
$$

where $\boldsymbol{X}_{i}^{t}$ and $\boldsymbol{V}_{i}^{t}$ denote the location and velocity vectors of the $i$ th particle at the $t$ th iteration; $\alpha, \beta_{l}$ and $\beta_{g}$ are user defined coefficients controlling the particle motion; $\gamma_{1}, \gamma_{2}$ and $\gamma_{3}$ are real random numbers between 0 and $1 ; \boldsymbol{P}_{i}$ is the best solution found for the $i$ th particle; $\boldsymbol{P}_{g}$ is the best solution in the entire population; $\gamma_{c}$ is the diversity preservation coefficient. A novel adaptive diversitypreservation technique was developed and implemented in the mixed-discrete PSO. For details of this algorithm, one is referred to [24].

Since the detailed data for the case studies in $[23,24]$, especially the characteristics data of the involved turbine types, is not publicly available, a direct comparison for the same cases used by Chowdhury et al. $[23,24]$ is not possible. Instead we construct a simplified version of the Horns Rev WF case that has been studied in this paper.

One major difference between the extended RS and the mixed-discrete PSO is about the number of WTs. Since the number of design variables (continuous and discrete) is assumed to be fixed for the mixed-discrete PSO, same as the other algorithms for mixed-integer-discrete optimization problems (such as differential evolution proposed in [37]), the number of WTs during the optimization process has to be fixed for the mixed-discrete PSO. However, such limitation doesn't exist for the extended $\mathrm{RS}$, as the number of WTs is allowed to change during the optimization process.

In order to compare the two algorithms, we consider the design optimization of non-uniform WFs with fixed number of turbines. To make things simpler, an ideal wind condition with constant wind speed $(8 \mathrm{~m} / \mathrm{s})$ blowing from west is assumed. The WF boundary, proximity constraints, the available turbine types and the LCOE modelling are the same as used before, but the constraint on the total capacity is excluded. The number of WTs is fixed as $N_{w t}=20$.

The same controlling parameters as used in the WF design optimization case study in [24] (see Table 1 in this reference) are used for the mixed-discrete PSO. The population size is set as 1200 according to the recommendation in [24] and 1000 iterations are allowed for one run. Thus, the total number of WF evaluations used in one run of the mixed-discrete PSO is 1200000. 
For the extended RS, the parameters used are: $N_{\text {repeat }}=9, E_{\text {max }}=100000, p_{a}=0$ and $p_{r}=0$. So each run of the extended RS consumes $100000 \mathrm{WF}$ evaluations. Note that by setting $p_{a}=0$ and $p_{r}=0$, the number of WTs will stay the same as the initial design, since only the locations and types of WTs will be changed during the optimization process.

For each of the 4 Scenarios, both algorithms are used for 10 runs, all using pure random starting designs as initial solutions. As described before, these initial solutions are generated randomly while satisfying all the constraints. The performance of these two algorithms is summarized in Table 5.

Table 5. Performance comparison between the extended RS and the mixed-discrete PSO

\begin{tabular}{clcccc}
\hline & \multicolumn{5}{c}{ LCOE [€/MWh] } \\
Scenario & & 1 & 2 & 3 & 4 \\
\hline \multirow{2}{*}{ Extended RS } & min & $\mathbf{1 0 3 . 9 8}$ & $\mathbf{1 0 2 . 8 2}$ & $\mathbf{1 0 7 . 2 9}$ & $\mathbf{1 0 1 . 2 4}$ \\
(10 runs) & mean & 104.46 & 103.46 & 107.45 & $\mathbf{1 0 1 . 2 4}$ \\
& $\max$ & 104.96 & 104.24 & 107.66 & $\mathbf{1 0 1 . 2 4}$ \\
\hline \multirow{2}{*}{$\begin{array}{c}\text { Mixed-discrete PSO } \\
\text { (10 runs) }\end{array}$} & $\min$ & 106.56 & 108.23 & 108.00 & 103.44 \\
& mean & 108.08 & 109.01 & 108.75 & 104.93 \\
& max & 109.20 & 110.85 & 109.60 & 106,68 \\
\hline
\end{tabular}

Clearly the extended RS outperforms the mixed-discrete PSO for this test case, as significantly lower LCOE values are achieved under all scenarios using the former algorithm, even if we compare the worst runs of the extended RS with the best runs of the mixed-discrete PSO. Considering the consumed number of WF evaluations for each run of the mixed-discrete PSO is more than 10 times larger than that of the extended RS, the superiority of the extended RS is more obvious.

Although for most of the scenarios, different runs of both algorithms converge to different results, the situation for the extended RS under Scenario 4 is different. Interestingly, the 10 runs of the extended RS all converge to the same final LCOE value. Examining the obtained designs in these 10 runs, we found that the designs are all composed of one type of WTs (LW2) but with different layouts. Other than that, the LCOE values of these 10 different designs are identical. This suggests that there are likely a group of global optimal solutions for this particular optimization problem that have the same LCOE value, and the extended RS could reach these optimal solutions with reasonable computational cost.

To further compare these two algorithms, the optimized design in the best run of the mixed-discrete PSO, the optimized design in one of the 10 runs of the extended RS, and the convergence histories for 10 runs of these two algorithms under Scenario 4 are shown in Figure 12. 


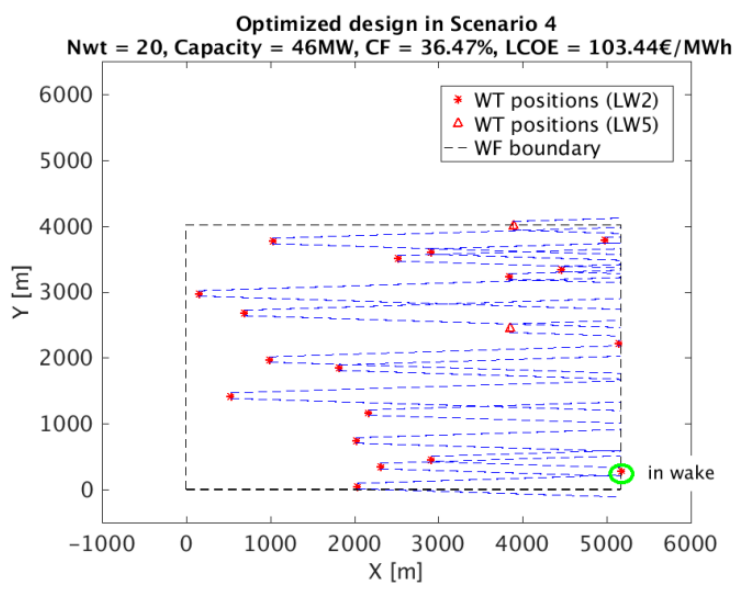

(a)

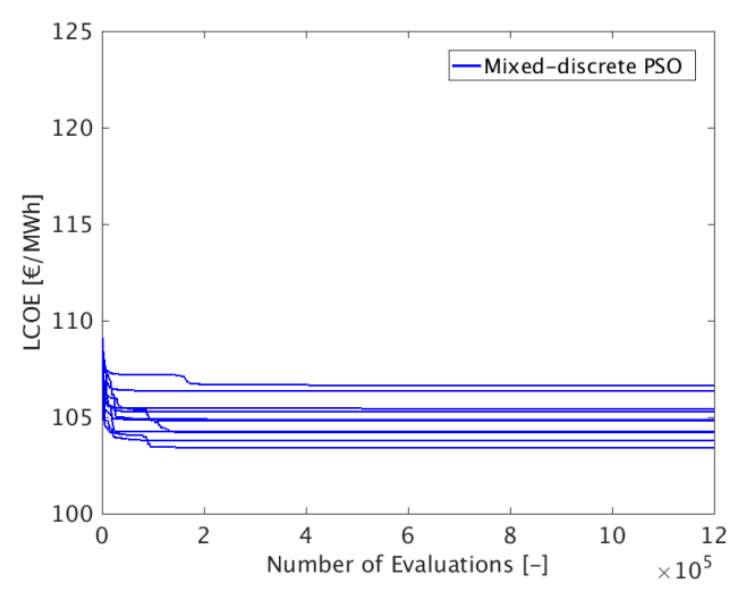

(c)

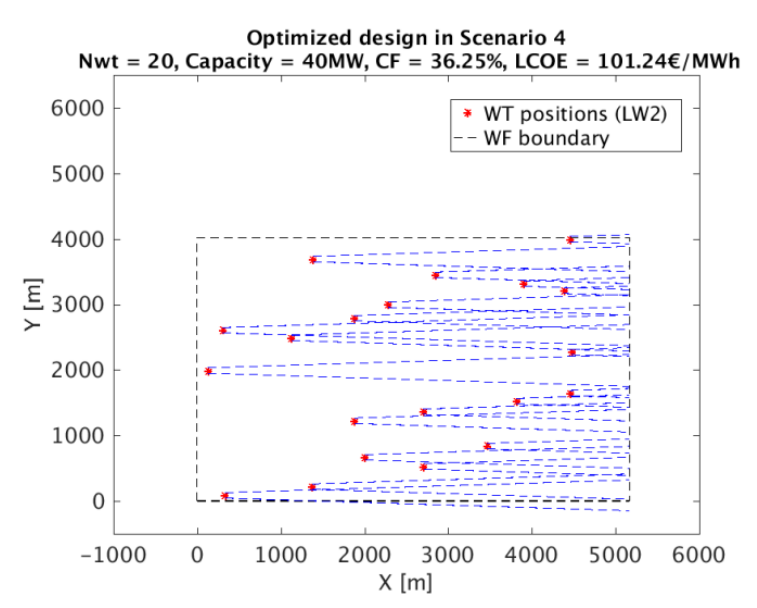

(b)

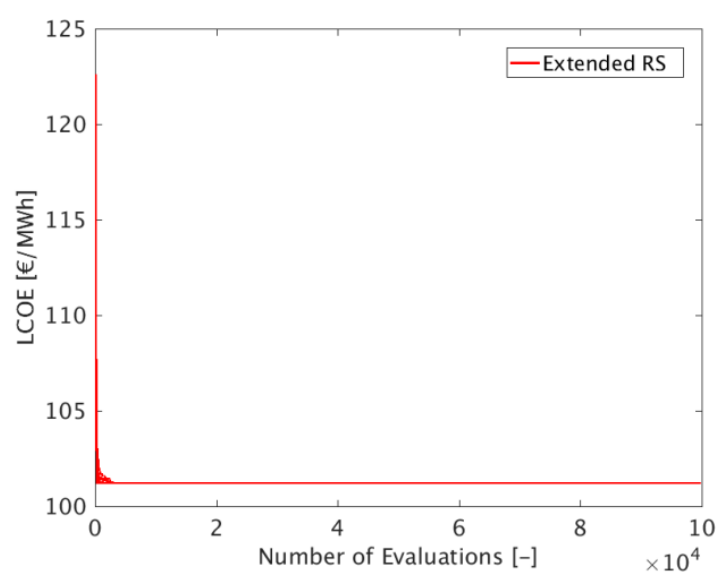

(d)

Figure 12. Optimized designs from (a) the best run of the mixed-discrete PSO and (b) one run of the extended RS, and convergence histories of 10 runs of (c) the mixed-discrete PSO and (d) the extended RS (blue dash lines in (a) and (b) represent wake zone boundaries from the 20 turbines)

Note that in Figure 12 (a) and (b), the boundaries of wake zones form each of the 20 WTs are represented by the blue dash lines. As we can see that the extended RS manages to find the optimal design that totally avoids the wake effect by smartly arranging the locations of WTs, while the mixeddiscrete PSO fails to do so. Examining the results of the other 9 runs of the extended RS reveals that the optimized designs are different but all avoid the wake effects, thus yield the same AEP and lead to the same LCOE. However, one should bear in mind that this is only achievable for this ideal wind condition (single wind direction). Avoiding wake effects completely is usually not possible for WFs under real wind conditions (such as shown in Figure 3). 
The convergence histories of both algorithm show that they both achieve most of the LCOE reductions in their initial stages of the optimization processes, while 10 different runs of the extended RS converge to the same LCOE value at a much faster speed.

Based on the above comparison study, we could safely conclude that the extended RS algorithm proposed in this study shows much better performance than the mixed-discrete PSO algorithm, at least for the considered test case.

\section{Conclusions}

In this paper the offshore WF design with WTs of different sizes has been considered. The results show, that for a given WF area, the optimization using larger WTs generally results in offshore WFs with a higher capacity factor, which is merely a result of their higher hub height (thus a higher wind speed) and less wake effects (due to the larger spacing between WTs). Whether using multiple types of WTs in an offshore WF, i.e., a non-uniform design, depends strongly on the difference of mean capital costs of different types of WTs. In the scenario where the capital cost per MW of small WTs is cheaper than that of the large WTs at a moderate degree (around 10\% in our study), the design with multiple types of WTs outperforms. Otherwise, the optimal offshore WF design will always be of a single type of WTs. Thus, the advantage of non-uniform design is quite dependent on the cost scenario, while its possible disadvantages in terms of supply chain management, installation, and O\&M are clearly presented, due to the inevitably increased level of complexity for these factors.

From the side of numerical optimization, it is always beneficial to formulate the problem as a nonuniform WF design optimization problem, since the proposed optimization method combined with multi-start strategy is capable of finding the final optimal design, whether it is uniform or non-uniform. Besides, the problem of uniform offshore WF design can be seen as a special case of the non-uniform one. Moreover, from the beginning of a WF project, it is typically not known whether uniform designs will outperform in reality, provided that more complex cost models are employed. In this case, it is also a better strategy to treat the problem as a general non-uniform design optimization problem. Hence, it is recommended to consider multiple types of WTs and treat the offshore WF as non-uniform in general at the design/planning stage.

Maintaining diversity of initial solutions is important for meta-heuristics. For the extended RS algorithm with multi-start strategy, it is thus required to strategically select the initial solutions. In context of design optimization of non-uniform WFs, it is always advised to include the initial solutions that are uniform, i.e., designs with single type of turbines.

The proposed optimization method, i.e., the extended RS algorithm, is a powerful method for designing non-uniform offshore WFs. Comparing to the mixed-discrete PSO algorithm, it is capable of 
considering unfixed number of turbines and can also found better designs while consuming much less WF evaluations.

The method developed in this study is a useful tool for WF design. It enables the WF developers/designers to further explore the feasible design space for offshore WFs and achieve even lower LCOE, by considering non-uniform design options using multiple types of WTs. Currently, only the turbine related design factors (type, number and location of WTs) are considered in the optimization process. It is envisaged that more complete design optimization involving other important design factors (e.g., hub-height, foundation, electrical system) could also be done by extending the current method and incorporating more realistic and advanced cost models. Our future work will investigate the WF design optimization problem along this direction.

\section{Acknowledgements}

This work was supported by the international collaboration project (DSF Sagsnr. 10-094544) under Danish Research and Innovation Council for Strategic Research, and The Energy Technology Development and Demonstration Program (EUDP J.nr. 64013-0405) under Danish Energy Agency. The authors would also like to sincerely thank and acknowledge the valuable comments and suggestions from the editor and the three anonymous reviewers.

\section{References}

[1] DONG Energy, The world's first offshore wind farm is retiring, (2017). http://www.dongenergy.com/en/media/newsroom/news/articles/the-worlds-first-offshore-windfarm-is-retiring (accessed March 22, 2017).

[2] GWEC, Global cumulative and annual offshore wind capacity 2011-2016, (2017). http://www.gwec.net/wp-content/uploads/2017/02/7_Annual-and-Global-CumulativeOffshore-wind-capacity-in-2016.jpg (accessed July 18, 2017).

[3] R. Perveen, N. Kishor, S.R. Mohanty, Off-shore wind farm development: Present status and challenges, Renewable and Sustainable Energy Reviews. 29 (2014) 780-792. doi:10.1016/j.rser.2013.08.108.

[4] M. Samorani, The Wind Farm Layout Optimization Problem, in: Handbook of Wind Power Systems, Springer-Verlag Berlin Heidelberg, 2013: pp. 21-38.

[5] G. Mosetti, C. Poloni, B. Diviacco, Optimization of wind turbine positioning in large windfarms by means of a genetic algorithm, Journal of Wind Engineering and Industrial 
Aerodynamics. 51 (1994) 105-116. doi:10.1016/0167-6105(94)90080-9.

[6] J. Herbert-Acero, O. Probst, P.-E. Réthoré, G. Larsen, K. Castillo-Villar, A review of methodological approaches for the design and optimization of wind farms, Energies. 7 (2014) 6930-7016. doi:10.3390/en7116930.

[7] P.S. Valverde, A.J.N.A. Sarmento, M. Alves, Offshore wind farm layout optimization - State of the art, in: Proceedings of the 2013 International Offshore and Polar Engineering Conference, Anchorage, Alaska, USA, 2013: pp. 157-163.

[8] C.N. Elkinton, J.F. Manwell, J.G. Mcgowan, Optimizing the layout of offshore wind energy systems, Marine Technology Society Journal. 42 (2008) 19-27.

[9] R.A. Rivas, J. Clausen, K.S. Hansen, L.E. Jensen, Solving the turbine positioning problem for large offshore wind farms by simulated annealing, Wind Engineering. 33 (2009) 287-297.

[10] J. González, M. Payan, J. Santos, A new and efficient method for optimal design of large offshore wind power plants, IEEE Transactions on Power Systems. 28 (2013) 3075-3084. http://ieeexplore.ieee.org/xpls/abs_all.jsp?arnumber=6502287.

[11] X. Gao, H. Yang, L. Lu, Study on offshore wind power potential and wind farm optimization in Hong Kong, Applied Energy. 130 (2014) 519-531. doi:10.1016/j.apenergy.2014.02.070.

[12] X. Gao, H. Yang, L. Lin, P. Koo, Wind turbine layout optimization using multi-population genetic algorithm and a case study in Hong Kong offshore, Journal of Wind Engineering and Industrial Aerodynamics. 139 (2015) 89-99. doi:10.1016/j.jweia.2015.01.018.

[13] S. Salcedo-Sanz, D. Gallo-Marazuela, a. Pastor-Sánchez, L. Carro-Calvo, A. Portilla-Figueras, L. Prieto, Offshore wind farm design with the Coral Reefs Optimization algorithm, Renewable Energy. 63 (2014) 109-115. doi:10.1016/j.renene.2013.09.004.

[14] J. Feng, W.Z. Shen, Solving the wind farm layout optimization problem using random search algorithm, Renewable Energy. 78 (2015) 182-192. doi:10.1016/j.renene.2015.01.005.

[15] X. Gao, H. Yang, L. Lu, Optimization of wind turbine layout position in a wind farm using a newly-developed two-dimensional wake model, Applied Energy. 174 (2016) 192-200. doi:10.1016/j.apenergy.2016.04.098.

[16] J. Feng, W.Z. Shen, Wind farm power production in the changing wind: Robustness quantification and layout optimization, Energy Conversion and Management. 148 (2017) 905914. doi:10.1016/j.enconman.2017.06.005.

[17] J. Serrano González, Á.L. Trigo García, M. Burgos Payán, J. Riquelme Santos, Á.G. González 
Rodríguez, Optimal wind-turbine micro-siting of offshore wind farms: A grid-like layout approach, Applied Energy. 200 (2017) 28-38. doi:10.1016/j.apenergy.2017.05.071.

[18] J. Park, K.H. Law, Layout optimization for maximizing wind farm power production using sequential convex programming, Applied Energy. 151 (2015) 320-334. doi:10.1016/j.apenergy.2015.03.139.

[19] D. Guirguis, D.A. Romero, C.H. Amon, Toward efficient optimization of wind farm layouts: Utilizing exact gradient information, Applied Energy. 179 (2016) 110-123. doi:10.1016/j.apenergy.2016.06.101.

[20] D. Guirguis, D.A. Romero, C.H. Amon, Gradient-based multidisciplinary design of wind farms with continuous-variable formulations, Applied Energy. 197 (2017) 279-291. doi:10.1016/j.apenergy.2017.04.030.

[21] P.Y. Zhang, D.A. Romero, J.C. Beck, C.H. Amon, Solving wind farm layout optimization with mixed integer programs and constraint programs, EURO Journal on Computational Optimization. 2 (2014) 195-219. doi:10.1007/s13675-014-0024-5.

[22] I. Mustakerov, D. Borissova, Wind turbines type and number choice using combinatorial optimization, Renewable Energy. 35 (2010) 1887-1894. doi:10.1016/j.renene.2009.12.012.

[23] S. Chowdhury, J. Zhang, A. Messac, L. Castillo, Optimizing the arrangement and the selection of turbines for wind farms subject to varying wind conditions, Renewable Energy. 52 (2013) 273-282. doi:10.1016/j.renene.2012.10.017.

[24] S. Chowdhury, W. Tong, A. Messac, J. Zhang, A mixed-discrete Particle Swarm Optimization algorithm with explicit diversity-preservation, Structural and Multidisciplinary Optimization. 47 (2013) 367-388. doi:10.1007/s00158-012-0851-z.

[25] N. Maslov, C. Claramunt, T. Wang, T. Tang, Method to estimate the visual impact of an offshore wind farm, Applied Energy. (2017). doi:10.1016/j.apenergy.2017.05.053.

[26] P.T. Madsen, M. Wahlberg, J. Tougaard, K. Lucke, P.L. Tyack, Wind turbine underwater noise and marine mammals: Implications of current knowledge and data needs, Marine Ecology Progress Series. 309 (2006) 279-295. doi:10.3354/meps309279.

[27] J. Feng, W.Z. Shen, C. Xu, Multi-Objective Random Search Algorithm for Simultaneously Optimizing Wind Farm Layout and Number of Turbines, Journal of Physics: Conference Series. 753 (2016) 32011. doi:10.1088/1742-6596/753/3/032011.

[28] P. Hou, W. Hu, M. Soltani, C. Chen, Z. Chen, Combined optimization for offshore wind 
turbine micro siting, Applied Energy. 189 (2017) 271-282.

doi:10.1016/j.apenergy.2016.11.083.

[29] A. Wedzik, T. Siewierski, M. Szypowski, A new method for simultaneous optimizing of wind farm's network layout and cable cross-sections by MILP optimization, Applied Energy. 182 (2016) 525-538. doi:10.1016/j.apenergy.2016.08.094.

[30] S. Lumbreras, A. Ramos, Offshore wind farm electrical design: A review, Wind Energy. 16 (2013) 459-473. doi:10.1002/we.1498.

[31] S. Chowdhury, J. Zhang, A. Messac, L. Castillo, Unrestricted wind farm layout optimization (UWFLO): Investigating key factors influencing the maximum power generation, Renewable Energy. 38 (2012) 16-30. doi:10.1016/j.renene.2011.06.033.

[32] L.L.P. Chamorro, N. Tobin, R.E.. E.A. Arndt, F. Sotiropoulos, Variable-sized wind turbines are a possibility for wind farm optimization, Wind Energy. 17 (2014) 1483-1494. doi:10.1002/we.1646.

[33] M. Abdulrahman, D. Wood, Investigating the Power-COE trade-off for wind farm layout optimization considering commercial turbine selection and hub height variation, Renewable Energy. 102 (2017) 267-278. doi:10.1016/j.renene.2016.10.038.

[34] Y. Chen, H. Li, K. Jin, Q. Song, Wind farm layout optimization using genetic algorithm with different hub height wind turbines, Energy Conversion and Management. 70 (2013) 56-65. doi:10.1016/j.enconman.2013.02.007.

[35] K. Chen, M.X. Song, X. Zhang, S.F. Wang, Wind turbine layout optimization with multiple hub height wind turbines using greedy algorithm, Renewable Energy. 96 (2016) 676-686. doi:10.1016/j.renene.2016.05.018.

[36] A. Vasel-Be-Hagh, C.L. Archer, Wind farm hub height optimization, Applied Energy. 195 (2017) 905-921. doi:10.1016/j.apenergy.2017.03.089.

[37] J.A. Lampinen, I. Zelinka, Mixed integer-discrete-continuous optimization by differential evolution, in: Proceedings of the 5th International Mendel Conference on Soft Computing, 1999: pp. 71-76. http://citeseerx.ist.psu.edu/viewdoc/summary?doi=10.1.1.35.9662.

[38] London Array Limited, How a turbine works, (2013). http://www.londonarray.com/how-aturbine-works/ (accessed March 25, 2017).

[39] MHI Vestas Offshore Wind, Burbo Bank Extension installation works completed within three months, (2016). http://www.mhivestasoffshore.com/bbe-installation-complete/ (accessed 
March 25, 2017).

[40] G. Sieros, P. Chaviaropoulos, J.D. Sørensen, B.H. Bulder, P. Jamieson, Upscaling wind turbines: theoretical and practical aspects and their impact on the cost of energy, Wind Energy. 15 (2012) 3-17. doi:10.1002/we.

[41] C. Desmond, J. Murphy, L. Blonk, W. Haans, Description of an 8 MW reference wind turbine, Journal of Physics: Conference Series. 753 (2016) 92013. doi:10.1088/17426596/753/9/092013.

[42] DTI, Study of the costs of offshore wind generation: A Report to the Renewables Advisory Board \& DTI, 2007.

[43] The Crown Estate, Offshore wind cost reduction pathways study, 2012. http://www.thecrownestate.co.uk/media/5462/ei-offshore-wind-operational-report-2015.pdf

[44] P. Heptonstall, R. Gross, P. Greenacre, T. Cockerill, The cost of offshore wind: Understanding the past and projecting the future, Energy Policy. 41 (2012) 815-821. doi:10.1016/j.enpol.2011.11.050.

[45] N. Ederer, Evaluating capital and operating cost efficiency of offshore wind farms: A DEA approach, Renewable and Sustainable Energy Reviews. 42 (2015) 1034-1046. doi:10.1016/j.rser.2014.10.071.

[46] Megavind, Megavind LCOE Model - Guidelines and documentation, 2015. https://megavind.windpower.org/download/2452/1500318_documentation_and_guidelinespdf.

[47] J. Manwell, J. McGowan, A. Rogers, Wind Energy Explained: Theory, Design, and Application, 2nd ed., John Wiely \& Sons, 2009.

[48] S.A. Grady, M.Y. Hussaini, M.M. Abdullah, Placement of wind turbines using genetic algorithms, Renewable Energy. 30 (2005) 259-270. doi:10.1016/j.renene.2004.05.007.

[49] C. Wan, J. Wang, G. Yang, X. Zhang, Optimal Micro-siting of Wind Farms by Particle Swarm Optimization, Advances in Swarm Intelligence. 6145 (2010) 198-205. http://www.springerlink.com/index/10.1007/978-3-642-13495-1.

[50] Z. Changshui, H. Guangdong, W. Jun, A fast algorithm based on the submodular property for optimization of wind turbine positioning, Renewable Energy. 36 (2011) 2951-2958. doi:10.1016/j.renene.2011.03.045.

[51] J. Zhang, S. Chowdhury, A. Messac, L. Castillo, A response surface-based cost model for wind farm design, Energy Policy. 42 (2012) 538-550. doi:10.1016/j.enpol.2011.12.021. 
[52] M. Dicorato, G. Forte, M. Pisani, M. Trovato, Guidelines for assessment of investment cost for offshore wind generation, Renewable Energy. 36 (2011) 2043-2051.

doi:10.1016/j.renene.2011.01.003.

[53] M. Shafiee, F. Brennan, I.A. Espinosa, A parametric whole life cost model for offshore wind farms, International Journal of Life Cycle Assessment. 21 (2016) 961-975. doi:10.1007/s11367-016-1075-z.

[54] P.E. Morthorst, L. Kitzing, Economics of building and operating offshore wind farms, in: Offshore Wind Farms: Technologies, Design and Operation, Elsevier Ltd, 2016: pp. 9-27. doi:10.1016/B978-0-08-100779-2.00002-7.

[55] A.G. Gonzalez-Rodriguez, Review of offshore wind farm cost components, Energy for Sustainable Development. 37 (2017) 10-19. doi:10.1016/j.esd.2016.12.001.

[56] P. Chaviaropoulos, A. Natarajan, INNWIND Deliverable D1.2.2: Definition of Performance Indicators (PIs) and Target Value, 2014.

[57] SETIS - TPWind, Key Performance Indicators for the European Wind Industrial Initiative, 2011.

[58] N.O. Jensen, A note on wind generator interaction, Roskile, Denmark, 1983. doi:Riso-M-2411.

[59] I. Katic, J. Højstrup, N.O. Jensen, A Simple Model for Cluster Efficiency, in: European Wind Energy Conference and Exhibition 1986, 1986: pp. 407-410.

[60] E.C. Morgan, M. Lackner, R.M. Vogel, L.G. Baise, Probability distributions for offshore wind speeds, Energy Conversion and Management. 52 (2011) 15-26.

doi:10.1016/j.enconman.2010.06.015.

[61] T. Burton, N. Jenkins, D. Sharpe, E. Bossanyi, Wind Energy Handbook, 2nd ed., John Wiley \& Sons, 2011 .

[62] J. Feng, W.Z. Shen, Modelling wind for wind farm layout optimization using joint distribution of wind speed and wind direction, Energies. 8 (2015) 3075-3092. doi:10.3390/en8043075.

[63] N.G. Nygaard, Wakes in very large wind farms and the effect of neighbouring wind farms, Journal of Physics: Conference Series. 524 (2014) 12162. doi:10.1088/1742$6596 / 524 / 1 / 012162$.

[64] N.G. Nygaard, S.D. Hansen, Wake effects between two neighbouring wind farms, Journal of Physics: Conference Series. 753 (2016) 32020. doi:10.1088/1742-6596/753/3/032020.

[65] M. Gendreau, J.-Y. Potvin, eds., Handbook of Metaheuristics, Second, Springer, 2010. 
[66] C. Blum, A. Roli, Metaheuristics in Combinatorial Optimization: Overview and Conceptual Comparison, ACM Computing Surveys. 35 (2003) 268-308. doi:10.1145/937503.937505.

[67] P. Diaz-Gomez, D. Hougen, Initial Population for Genetic Algorithms: A Metric Approach., in: Proceedings of the 2007 International Conference on Genetic and Evolutionary Methods, 2007: pp. 43-49.

[68] B. Saavedra-Moreno, S. Salcedo-Sanz, A. Paniagua-Tineo, L. Prieto, A. Portilla-Figueras, Seeding evolutionary algorithms with heuristics for optimal wind turbines positioning in wind farms, Renewable Energy. 36 (2011) 2838-2844. doi:10.1016/j.renene.2011.04.018.

[69] R. Martí, M.G.C. Resende, C.C. Ribeiro, Multi-start methods for combinatorial optimization, European Journal of Operational Research. 226 (2013) 1-8. doi:10.1016/j.ejor.2012.10.012.

\section{Appendix}

Table A1. Power and thrust parameters of the three types of WTs

\begin{tabular}{cccccccc}
\hline \multirow{2}{*}{ Wind speed $[\mathrm{m} / \mathrm{s}]$} & \multicolumn{3}{c}{ Power $[\mathrm{kW}]$} & \multicolumn{5}{c}{ Thrust $[\mathrm{kN}]$} \\
& LW2 & LW5 & LW8 & LW2 & LW5 & LW8 & Ct $[-]$ \\
\hline 4.0 & 28 & 69 & 110 & 48 & 119 & 190 & 0.92 \\
4.5 & 88 & 219 & 350 & 58 & 145 & 232 & 0.88 \\
5.0 & 150 & 375 & 600 & 68 & 171 & 273 & 0.85 \\
5.5 & 213 & 531 & 850 & 81 & 203 & 324 & 0.83 \\
6.0 & 285 & 713 & 1140 & 95 & 238 & 381 & 0.82 \\
6.5 & 373 & 931 & 1490 & 110 & 275 & 440 & 0.81 \\
7.0 & 475 & 1188 & 1900 & 126 & 316 & 505 & 0.80 \\
7.5 & 593 & 1481 & 2370 & 143 & 358 & 573 & 0.79 \\
8.0 & 725 & 1813 & 2900 & 162 & 405 & 648 & 0.78 \\
8.5 & 875 & 2188 & 3500 & 181 & 452 & 723 & 0.77 \\
9.0 & 1039 & 2597 & 4155 & 200 & 500 & 800 & 0.76 \\
9.5 & 1218 & 3044 & 4870 & 219 & 548 & 876 & 0.75 \\
10.0 & 1408 & 3519 & 5630 & 236 & 591 & 945 & 0.73 \\
10.5 & 1605 & 4013 & 6420 & 254 & 634 & 1014 & 0.71 \\
11.0 & 1788 & 4469 & 7150 & 263 & 658 & 1052 & 0.67 \\
11.5 & 1903 & 4756 & 7610 & 257 & 643 & 1028 & 0.60 \\
12.0 & 1966 & 4916 & 7865 & 243 & 608 & 972 & 0.52 \\
12.5 & 1985 & 4963 & 7940 & 226 & 566 & 905 & 0.45 \\
13.0 & 1993 & 4981 & 7970 & 212 & 529 & 847 & 0.39 \\
13.5 & 2000 & 5000 & 8000 & 200 & 501 & 801 & 0.34 \\
14.0 & 2000 & 5000 & 8000 & 191 & 478 & 765 & 0.30 \\
14.5 & 2000 & 5000 & 8000 & 183 & 456 & 730 & 0.27 \\
15.0 & 2000 & 5000 & 8000 & 175 & 438 & 700 & 0.24 \\
15.5 & 2000 & 5000 & 8000 & 167 & 418 & 668 & 0.22 \\
16.0 & 2000 & 5000 & 8000 & 161 & 403 & 644 & 0.19 \\
\hline
\end{tabular}




\begin{tabular}{llllllll}
\hline 16.5 & 2000 & 5000 & 8000 & 156 & 390 & 624 & 0.18 \\
17.0 & 2000 & 5000 & 8000 & 151 & 378 & 604 & 0.16 \\
17.5 & 2000 & 5000 & 8000 & 147 & 367 & 587 & 0.15 \\
18.0 & 2000 & 5000 & 8000 & 143 & 357 & 571 & 0.14 \\
18.5 & 2000 & 5000 & 8000 & 139 & 348 & 557 & 0.13 \\
19.0 & 2000 & 5000 & 8000 & 136 & 339 & 542 & 0.12 \\
19.5 & 2000 & 5000 & 8000 & 132 & 330 & 528 & 0.11 \\
20.0 & 2000 & 5000 & 8000 & 129 & 323 & 516 & 0.10 \\
20.5 & 2000 & 5000 & 8000 & 126 & 316 & 505 & 0.09 \\
21.0 & 2000 & 5000 & 8000 & 124 & 311 & 497 & 0.09 \\
21.5 & 2000 & 5000 & 8000 & 122 & 304 & 486 & 0.08 \\
22.0 & 2000 & 5000 & 8000 & 119 & 298 & 476 & 0.08 \\
22.5 & 2000 & 5000 & 8000 & 118 & 295 & 472 & 0.07 \\
23.0 & 2000 & 5000 & 8000 & 115 & 288 & 461 & 0.07 \\
23.5 & 2000 & 5000 & 8000 & 114 & 284 & 454 & 0.06 \\
24.0 & 2000 & 5000 & 8000 & 111 & 278 & 445 & 0.06 \\
24.5 & 2000 & 5000 & 8000 & 111 & 276 & 442 & 0.06 \\
25.0 & 2000 & 5000 & 8000 & 109 & 273 & 437 & 0.05 \\
\hline
\end{tabular}

Note: data for LW8 from [41], data for LW2 and LW5 derived from LW8. 